CRYSTALLOGRAPHIC COMMUNICATIONS

ISSN 2056-9890

Received 29 December 2016

Accepted 21 February 2017

Edited by M. Weil, Vienna University of Technology, Austria

Keywords: crystal structure; crystal diffraction; gallium; borate.

CCDC reference: 1534100

Supporting information: this article has supporting information at journals.iucr.org/e

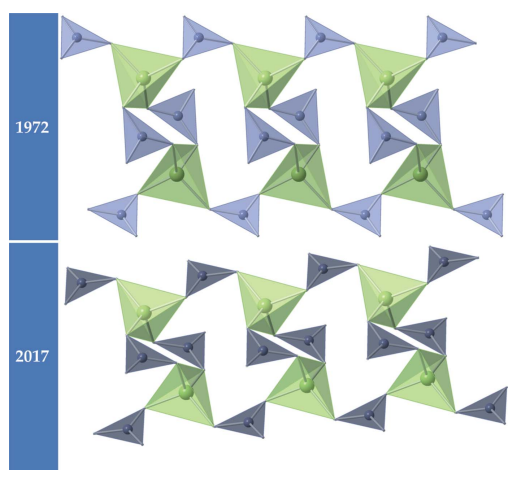

OPEN $\odot$ ACCESS

\section{Crystal structure of $\mathrm{Li}_{3} \mathrm{Ga}\left(\mathrm{BO}_{3}\right)_{2}$}

\author{
Robert W. Smith, ${ }^{\mathrm{a} *}$ Darien Holman $^{\mathrm{a}}$ and Eric M. Villa ${ }^{\mathrm{b}}$
}

${ }^{\mathrm{a}}$ Department of Chemistry, University of Nebraska at Omaha, 6001 Dodge Street, Omaha, Nebraska 68182, USA, and ${ }^{\mathbf{b}}$ Department of Chemistry, Creighton University, 2500 California Plaza, Omaha, Nebraska 68178, USA. *Correspondence e-mail: robertsmith@unomaha.edu

The crystal structure of trilithium gallium bis(orthoborate), $\mathrm{Li}_{3} \mathrm{Ga}\left(\mathrm{BO}_{3}\right)_{2}$, is isotypic with $\mathrm{Li}_{3} \mathrm{Al}\left(\mathrm{BO}_{3}\right)_{2}$ in a triclinic cell in space-group type $P \overline{1}$. The three $\mathrm{Li}$ and the unique $\mathrm{Ga}$ atom are coordinated by four $\mathrm{O}$ atoms each in tetrahedra, and the two $\mathrm{B}$ atoms are coordinated by three $\mathrm{O}$ atoms in orthoborate triangles. Chains with composition $\left[\mathrm{Ga}_{2}\left(\mathrm{BO}_{3}\right)_{4}\right]^{6-}$ extend along the $a$ axis. The $\mathrm{Li}$ atoms interleave these chains in tetrahedral interstices. A comparison is made between the structure model of the title compound and that of a previously reported model for a compound with the same composition [Abdullaev \& Mamedov (1972). Zh. Strukt. Khim. 13, 943-946.]

\section{Chemical context}

We are examining the alkali metal/gallium/borate phase diagrams, investigations of which have revealed to date, among others, the homologous series $A_{2} \mathrm{Ga}_{2} \mathrm{O}\left(\mathrm{BO}_{3}\right)_{2}$, in which $A=\mathrm{Na}, \mathrm{K}, \mathrm{Rb}$, and Cs (Corbel \& Leblanc, 2000; Smith, 1995; Smith et al., 1997,2008, respectively) and the homologous series $A_{3} \mathrm{Ga}\left(\mathrm{BO}_{3}\right)_{2}$, in which $A=\mathrm{Li}, \mathrm{Na}, \mathrm{K}, \mathrm{Rb}$, and Cs. We report herein the crystal structure of the lithium analog (Fig. 1) of the latter series, which is the only one which melts congruently, which has a unique structure among the series, and which is isotypic with $\mathrm{Li}_{3} \mathrm{Al}\left(\mathrm{BO}_{3}\right)_{2}$ ( $\mathrm{He}$ et al., 2002); the other analogs have yet to be crystallized in the form of single crystals, but are structurally distinct from the lithium analog and isotypic with each other based on their powder X-ray diffraction patterns.

A crystal structure for this compound was previously reported by Abdullaev \& Mamedov (1972) in the same triclinic space-group type $P \overline{1}$, and with the same galliumborate polyhedral pattern but with important differences with the structure reported herein, to wit: slightly different cell parameters and a different reduced cell, a significantly smaller cell volume (i.e., $3 \%$ smaller), less regular bond-valence sums (BVS), greater deviations from expected interatomic distances, and irregular, five- and six-coordinate lithiumcentered polyhedra. Table 1 compares interatomic distances from the structure reported by Addullaev \& Mamedov (1972) and this report, with expected distances using Shannon's radii (Shannon, 1976); it also lists bond-valence sums for each structure. We have considered as bonds all $\mathrm{Li}-\mathrm{O}$ distances under $3 \AA$ from the 1972 report because doing so produces more reasonable BVS values, thus rendering some of the lithium atoms as being five- or six-coordinate in the previous structure report. It should be noted that the authors, however, reported all lithium atoms as tetrahedrally coordinated. The present structure model clearly differs from the 1972 structure 
Table 1

Comparison of the two structures with composition $\mathrm{Li}_{3} \mathrm{Ga}\left(\mathrm{BO}_{3}\right)_{2}$.

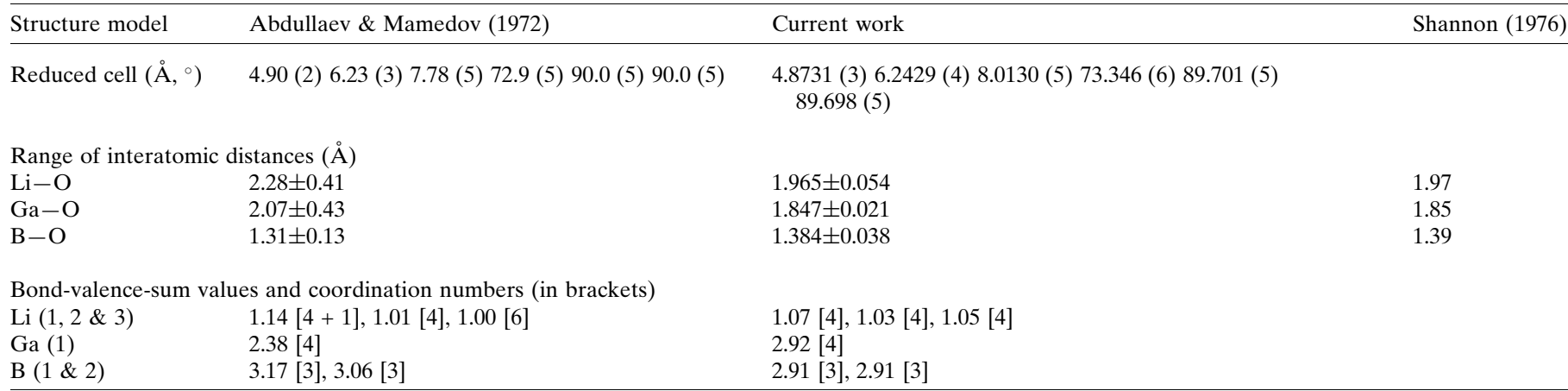

model and hence indicates a second possible modification for this composition. Whether a polymorphic relation exists between the two phases remains unknown and needs additional proof by using complementary methods such as thermal analysis.

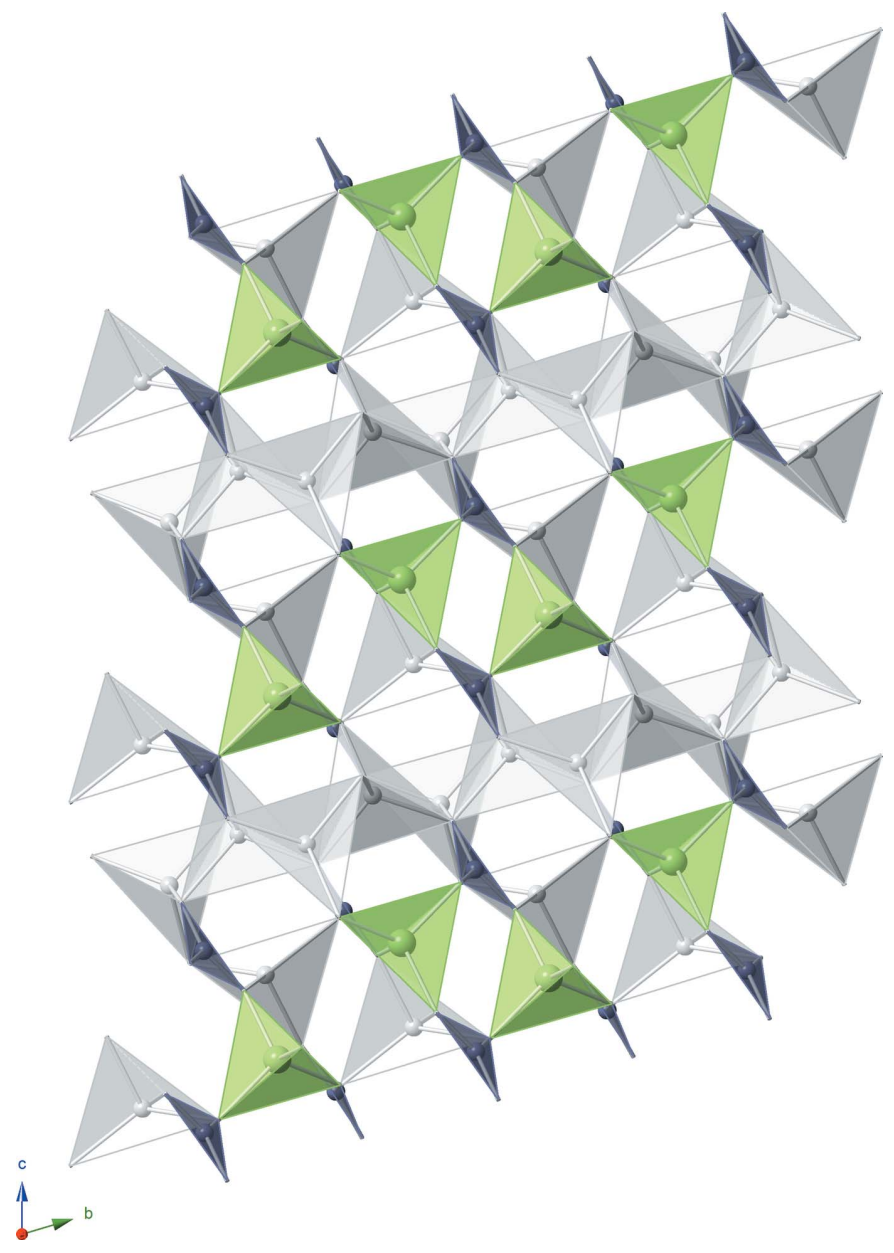

Figure 1

A projection of the crystal structure of $\mathrm{Li}_{3} \mathrm{Ga}\left(\mathrm{BO}_{3}\right)_{2}$ along the $a$ axis. Infinite $\left[\mathrm{Ga}_{2}\left(\mathrm{BO}_{3}\right)_{4}\right]^{6-}$ chains are linked together by sheets of Li atoms in tetrahedral voids. $\mathrm{GaO}_{4}$ tetrahedra are light green, $\mathrm{LiO}_{4}$ tetrahedra are light gray, and $\mathrm{BO}_{3}$ triangles are blue-grey. Corner $\mathrm{O}$ atoms have been omitted for clarity.

\section{Structural commentary}

The crystal structure of the title compound consists of lithiumand gallium-centered tetrahedra and boron-centered triangles, all of which share oxygen vertices (Fig.1). Each $\mathrm{GaO}_{4}$ tetrahedron is linked to four $\mathrm{BO}_{3}$ triangles and six $\mathrm{LiO}_{4}$ tetrahedra. The gallium-centered tetrahedra and boron-centered triangles adjoin through shared vertices to form infinite chains of composition $\left[\mathrm{Ga}_{2}\left(\mathrm{BO}_{3}\right)_{4}\right]^{6-}$, with the chains extending parallel to the $a$ axis; lithium cations interleave the chains in tetrahedral interstices. Fig. 2 shows a comparison of the galliumborate chains in both the previously reported structure (Abdullaev \& Mamedov, 1972) and the structure presented here. The two exhibit the same connectivity but have sizeable

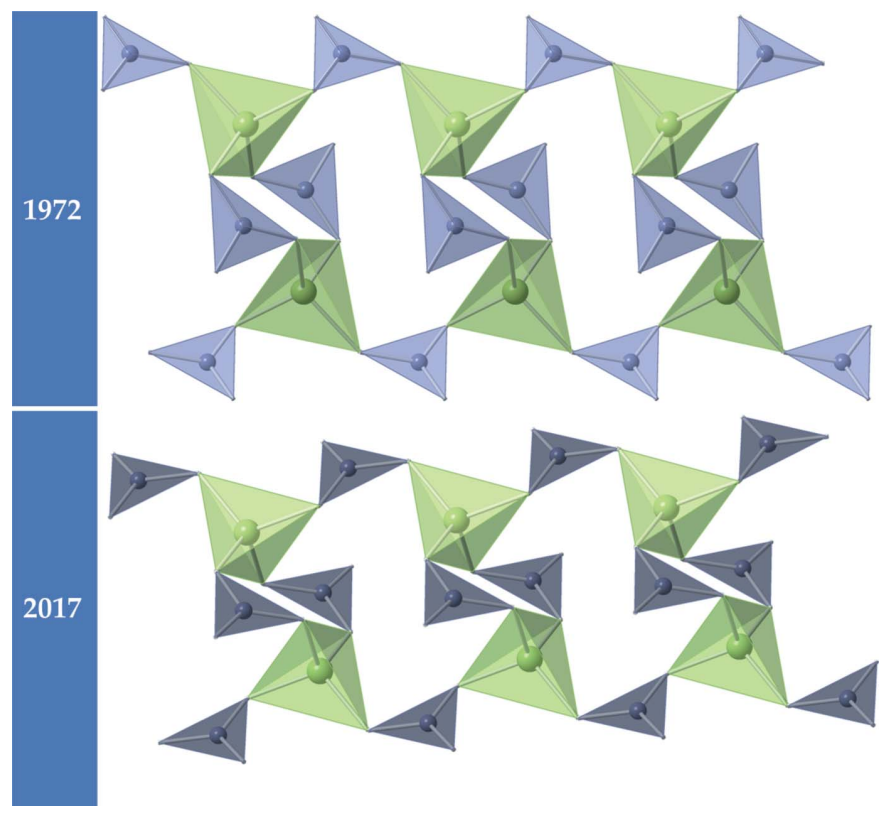

Figure 2

A comparison of the infinite $\left[\mathrm{Ga}_{2}\left(\mathrm{BO}_{3}\right)_{4}\right]^{6-}$ chains in the structures of $\mathrm{Li}_{3} \mathrm{Ga}\left(\mathrm{BO}_{3}\right)_{2}$ with respect to the model by Abdullaev \& Mamedov (1972) (top) and the structure model presented in this manuscript. The connectivity of these two chains are the same but there are important differences in the actual bonding. In our structure model, these chains run down the $c$ axis. Here the $\mathrm{GaO}_{4}$ tetrahedra are light green and the $\mathrm{BO}_{3}$ triangles are blue-grey (which are shown in light blue-grey in the 1972 structure). Corner $\mathrm{O}$ atoms have been omitted for clarity. 


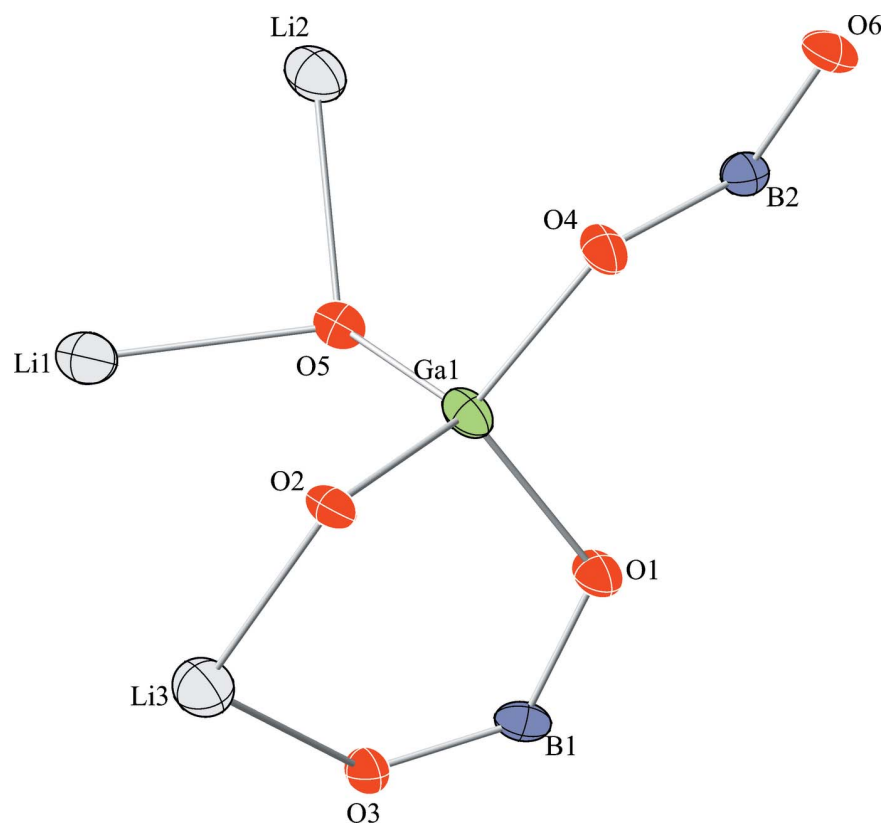

Figure 3

The asymmetric unit of the title structure with atom labelling. Displacement ellipsoids are drawn at the $75 \%$ probability level.

differences in bond lengths, bond angles and bond-valencesum values (see Table 1). Averaged interatomic distances for the title structure are consistent with those determined from the ionic radii reported by Shannon (1976), viz. 1.97 (5), 1.85 (2), and 1.39 (4) $\AA$ for the experimentally determined $\mathrm{Li}-\mathrm{O}, \mathrm{Ga}-\mathrm{O}$, and $\mathrm{B}-\mathrm{O}$ distances, respectively. We also calculated the bond-valence-um values for each element using the values provided by Brese \& O'Keeffe (1991). The results (Table 1) are in good agreement with the expected values of 1 , 3, 3 and 2 for $\mathrm{Li}, \mathrm{Ga}, \mathrm{B}$ and $\mathrm{O}$ atoms, respectively.

Lastly, Fig. 3 displays the anisotropic displacement parameters of the atoms within the asymmetric unit of the title structure.

\section{Synthesis and crystallization}

Powder samples were made by solid-state reactions starting with stoichiometric proportions of lithium nitrate, gallium(III) nitrate, and boric acid. We first ground the starting materials and fired them in an alumina crucible at $573 \mathrm{~K}$ for two $\mathrm{h}$ to decompose them to finely divided oxides, after which we progressively heated the samples to $973 \mathrm{~K}$ at 50 to $100 \mathrm{~K}$ and 24-hour increments, grinding the samples between each successive heat treatment. Samples were single-phase as revealed by powder X-ray diffraction.

Single crystals were grown from the melt. About $500 \mathrm{mg}$ of sample were placed in a platinum dish, heated to $1033 \mathrm{~K}$ in a box oven, slow-cooled at $10 \mathrm{~K} \mathrm{~h}^{-1}$ to about $470 \mathrm{~K}$, and then air-quenched. Several small, clear, colorless crystals were physically removed from the platinum crucible and mounted on a goniometer for a preliminary scan in order to find one of suitable quality.
Table 2

Experimental details.

\begin{tabular}{|c|c|}
\hline \multicolumn{2}{|l|}{ Crystal data } \\
\hline Chemical formula & $\mathrm{Li}_{3} \mathrm{Ga}\left(\mathrm{BO}_{3}\right)_{2}$ \\
\hline$M_{\mathrm{r}}$ & 208.16 \\
\hline Crystal system, space group & Triclinic, $P \overline{1}$ \\
\hline Temperature (K) & 293 \\
\hline$a, b, c(\AA)$ & $4.8731(3), 6.2429(4), 8.0130(5)$ \\
\hline$\alpha, \beta, \gamma\left({ }^{\circ}\right)$ & $73.346(6), 89.701(5), 89.698(5)$ \\
\hline$V\left(\AA^{3}\right)$ & $233.54(3)$ \\
\hline$Z$ & 2 \\
\hline Radiation type & Мо $K \alpha$ \\
\hline$\mu\left(\mathrm{mm}^{-1}\right)$ & 5.84 \\
\hline Crystal size $(\mathrm{mm})$ & $0.09 \times 0.03 \times 0.01$ \\
\hline \multicolumn{2}{|l|}{ Data collection } \\
\hline Diffractometer & Rigaku SCX mini diffractometer \\
\hline Absorption correction & $\begin{array}{l}\text { Multi-scan (CrysAlis PRO; Rigaku } \\
\text { Oxford Diffraction, 2015) }\end{array}$ \\
\hline$T_{\min }, T_{\max }$ & $0.785,1.000$ \\
\hline $\begin{array}{l}\text { No. of measured, independent and } \\
\text { observed }[I>2 \sigma(I)] \text { reflections }\end{array}$ & $2936,1397,1265$ \\
\hline$R_{\text {int }}$ & 0.029 \\
\hline$(\sin \theta / \lambda)_{\max }\left(\AA^{-1}\right)$ & 0.714 \\
\hline \multicolumn{2}{|l|}{ Refinement } \\
\hline$R\left[F^{2}>2 \sigma\left(F^{2}\right)\right], w R\left(F^{2}\right), S$ & $0.028,0.067,1.09$ \\
\hline No. of reflections & 1397 \\
\hline No. of parameters & 109 \\
\hline$\Delta \rho_{\max }, \Delta \rho_{\min }\left(\mathrm{e} \AA^{-3}\right)$ & $1.04,-0.76$ \\
\hline
\end{tabular}

Computer programs: CrysAlis PRO (Rigaku Oxford Diffraction, 2015), SHELXT (Sheldrick, 2015a), SHELXL2014 (Sheldrick, 2015b) and OLEX2 (Dolomanov et al., 2009).

\section{Refinement}

Crystal data, data collection and structure refinement details are summarized in Table 2.

\section{Acknowledgements}

EMV would like to acknowledge Creighton University for financial support.

\section{References}

Abdullaev, G. K. \& Mamedov, Kh. S. (1972). Zh. Strukt. Khim. 13, 943-946.

Brese, N. E. \& O'Keeffe, M. (1991). Acta Cryst. B47, 192-197.

Corbel, G. \& Leblanc, M. (2000). J. Solid State Chem. 154, 344-349.

Dolomanov, O. V., Bourhis, L. J., Gildea, R. J., Howard, J. A. K. \& Puschmann, H. (2009). J. Appl. Cryst. 42, 339-341.

He, M., Chen, X. L., Gramlich, V., Baerlocher, Ch., Zhou, T. \& Hu, B. Q. (2002). J. Solid State Chem. 163, 369-376.

Rigaku Oxford Diffraction (2015). CrysAlis PRO. Rigaku Oxford Diffraction, Yarnton, Oxfordshire, England.

Shannon, R. D. (1976). Acta Cryst. A32, 751-767.

Sheldrick, G. M. (2015a). Acta Cryst. A71, 3-8.

Sheldrick, G. M. (2015b). Acta Cryst. C71, 3-8.

Smith, R. W. (1995). Acta Cryst. C51, 547-549.

Smith, R. W., Hu, C. \& DeSpain, C. D. (2008). Acta Cryst. E64, i23.

Smith, R. W., Kennard, M. A. \& Dudik, M. J. (1997). Mater. Res. Bull. 32, 649-656. 


\section{supporting information}

Acta Cryst. (2017). E73, 456-458 [https://doi.org/10.1107/S205698901700295X]

\section{Crystal structure of $\mathrm{Li}_{3} \mathrm{Ga}\left(\mathrm{BO}_{3}\right)_{2}$}

\section{Robert W. Smith, Darien Holman and Eric M. Villa}

\section{Computing details}

Data collection: CrysAlis PRO (Rigaku Oxford Diffraction, 2015); cell refinement: CrysAlis PRO (Rigaku Oxford Diffraction, 2015); data reduction: CrysAlis PRO (Rigaku Oxford Diffraction, 2015); program(s) used to solve structure: SHELXT (Sheldrick, 2015a); program(s) used to refine structure: SHELXL2014 (Sheldrick, 2015b); molecular graphics: OLEX2 (Dolomanov et al., 2009); software used to prepare material for publication: OLEX2 (Dolomanov et al., 2009).

Trilithium gallium bis(orthoborate)

Crystal data

$\mathrm{Li}_{3} \mathrm{Ga}\left(\mathrm{BO}_{3}\right)_{2}$

$M_{r}=208.16$

Triclinic, $P \overline{1}$

$a=4.8731(3) \AA$

$b=6.2429(4) \AA$

$c=8.0130(5) \AA$

$\alpha=73.346(6)^{\circ}$

$\beta=89.701(5)^{\circ}$

$\gamma=89.698(5)^{\circ}$

$V=233.54(3) \AA^{3}$

\section{Data collection}

Rigaku SCX mini diffractometer

Radiation source: fine-focus sealed X-ray tube, Enhance (Mo) X-ray Source

Graphite monochromator

$\omega$ scans

Absorption correction: multi-scan

(CrysAlis PRO; Rigaku Oxford Diffraction, 2015)

\section{Refinement}

Refinement on $F^{2}$

Least-squares matrix: full

$R\left[F^{2}>2 \sigma\left(F^{2}\right)\right]=0.028$

$w R\left(F^{2}\right)=0.067$

$S=1.09$

1397 reflections

109 parameters
$Z=2$

$F(000)=196$

$D_{\mathrm{x}}=2.960 \mathrm{Mg} \mathrm{m}^{-3}$

Mo $K \alpha$ radiation, $\lambda=0.71073 \AA$

Cell parameters from 1687 reflections

$\theta=2.7-32.5^{\circ}$

$\mu=5.84 \mathrm{~mm}^{-1}$

$T=293 \mathrm{~K}$

Plate, clear light colourless

$0.09 \times 0.03 \times 0.01 \mathrm{~mm}$

$T_{\min }=0.785, T_{\max }=1.000$

2936 measured reflections

1397 independent reflections

1265 reflections with $I>2 \sigma(I)$

$R_{\text {int }}=0.029$

$\theta_{\max }=30.5^{\circ}, \theta_{\min }=2.7^{\circ}$

$h=-6 \rightarrow 6$

$k=-8 \rightarrow 8$

$l=-11 \rightarrow 11$

0 restraints

Primary atom site location: dual

$w=1 /\left[\sigma^{2}\left(F_{\mathrm{o}}^{2}\right)+(0.0282 P)^{2}+0.1042 P\right]$

where $P=\left(F_{\mathrm{o}}{ }^{2}+2 F_{\mathrm{c}}{ }^{2}\right) / 3$

$(\Delta / \sigma)_{\max }<0.001$

$\Delta \rho_{\max }=1.04 \mathrm{e}^{-3}$

$\Delta \rho_{\min }=-0.76$ e $\AA^{-3}$ 


\section{Special details}

Geometry. All esds (except the esd in the dihedral angle between two 1.s. planes) are estimated using the full covariance matrix. The cell esds are taken into account individually in the estimation of esds in distances, angles and torsion angles; correlations between esds in cell parameters are only used when they are defined by crystal symmetry. An approximate (isotropic) treatment of cell esds is used for estimating esds involving l.s. planes.

Refinement. Crystals of $\mathrm{Li}_{3} \mathrm{Ga}\left(\mathrm{BO}_{3}\right)_{2}$ were mounted on MiTeGen Microloop with non-drying immersion oil. The crystal was then optically aligned on the Rigaku SCX-Mini diffractometer using a digital camera. Initial matrix images were collected to determine the unit cell, validity and proper exposure time. Three hemispheres (where $\varphi=0.0,120.0$ and 240.0) of data were collected with each consisting 180 images each with $1.00^{\circ}$ widths and a $1.00^{\circ}$ step. The structure of $\mathrm{Li}_{3} \mathrm{Ga}\left(\mathrm{BO}_{3}\right)_{2}$ was refined using SHELXT (Sheldrick, 2015) Intrinsic Phasing and SHELXL (Sheldrick, 2015). Olex2 (Dolomanov et al., 2009) was used as a graphical interface. Images of the above compound were made using CrystalMaker for Windows, version 9.2.8. The refinement proceeded without any incidents and without any need for modelling disorder or twinning or any constraints or restraints.

Refinement of the structure was based on $\mathrm{F}^{2}$ against all reflections. The $\mathrm{R}$-factor $\mathrm{R}$ is based on $\mathrm{F}^{2}>2$ ? $\left(\mathrm{F}^{2}\right)$, but is not relevant to the choice of reflections for refinement; whereas the weighted R-factor $\mathrm{wR}$ and goodness of fit $\mathrm{S}$ are based on $\mathrm{F}^{2}$. The maximum electron denisty is 1.035 and is located $1.629 \AA$ from $\mathrm{Li}(2), 1.960 \AA$ from $\operatorname{Li}(1)$ and $2.169 \AA$ from a different $\operatorname{Li}(1)$, which leads to nothing reasonable. All other maximum peaks are under 0.600 .

Fractional atomic coordinates and isotropic or equivalent isotropic displacement parameters $\left(\AA^{2}\right)$

\begin{tabular}{lllll}
\hline & $x$ & $y$ & $z$ & $U_{\text {iso }} * / U_{\text {eq }}$ \\
\hline Ga1 & $0.65937(6)$ & $0.22951(4)$ & $0.60906(4)$ & $0.00968(10)$ \\
B1 & $0.1691(6)$ & $0.0135(5)$ & $0.7454(4)$ & $0.0088(5)$ \\
B2 & $0.6631(6)$ & $0.5014(5)$ & $0.2489(4)$ & $0.0094(5)$ \\
O1 & $0.3037(4)$ & $0.1391(3)$ & $0.5958(2)$ & $0.0116(4)$ \\
O2 & $-0.1202(4)$ & $-0.0028(3)$ & $0.7317(2)$ & $0.0101(4)$ \\
O3 & $0.2978(4)$ & $-0.0830(3)$ & $0.8955(2)$ & $0.0109(4)$ \\
O4 & $0.7953(4)$ & $0.3547(3)$ & $0.3900(2)$ & $0.0117(4)$ \\
O5 & $0.3833(4)$ & $0.5529(3)$ & $0.2727(2)$ & $0.0100(4)$ \\
O6 & $0.7921(4)$ & $0.5853(3)$ & $0.0967(2)$ & $0.0120(4)$ \\
Li1 & $0.1828(10)$ & $-0.3717(8)$ & $1.0414(6)$ & $0.0154(10)$ \\
Li2 & $1.1645(10)$ & $0.2738(8)$ & $0.3670(6)$ & $0.0143(9)$ \\
Li3 & $0.6839(10)$ & $0.8785(8)$ & $-0.0399(6)$ & $0.0159(10)$ \\
\hline
\end{tabular}

Atomic displacement parameters $\left(\AA^{2}\right)$

\begin{tabular}{lllllll}
\hline & $U^{11}$ & $U^{22}$ & $U^{33}$ & $U^{12}$ & $U^{13}$ & $U^{23}$ \\
\hline Ga1 & $0.00877(15)$ & $0.01034(15)$ & $0.00789(15)$ & $-0.00023(10)$ & $0.00130(10)$ & $0.00064(10)$ \\
B1 & $0.0103(13)$ & $0.0052(11)$ & $0.0106(12)$ & $0.0008(9)$ & $0.0021(10)$ & $-0.0018(10)$ \\
B2 & $0.0091(13)$ & $0.0088(12)$ & $0.0095(12)$ & $-0.0013(10)$ & $0.0002(10)$ & $-0.0013(10)$ \\
O1 & $0.0098(9)$ & $0.0134(9)$ & $0.0090(8)$ & $-0.0028(7)$ & $0.0011(7)$ & $0.0012(7)$ \\
O2 & $0.0068(8)$ & $0.0105(8)$ & $0.0098(8)$ & $0.0013(6)$ & $0.0002(7)$ & $0.0020(7)$ \\
O3 & $0.0107(9)$ & $0.0104(8)$ & $0.0094(8)$ & $-0.0007(7)$ & $-0.0018(7)$ & $0.0008(7)$ \\
O4 & $0.0110(9)$ & $0.0138(9)$ & $0.0066(8)$ & $0.0017(7)$ & $0.0012(7)$ & $0.0030(7)$ \\
O5 & $0.0088(9)$ & $0.0102(8)$ & $0.0104(8)$ & $0.0008(7)$ & $0.0013(7)$ & $-0.0017(7)$ \\
O6 & $0.0136(9)$ & $0.0114(9)$ & $0.0088(8)$ & $-0.0001(7)$ & $0.0029(7)$ & $0.0005(7)$ \\
Li1 & $0.019(2)$ & $0.013(2)$ & $0.013(2)$ & $-0.0038(18)$ & $0.0013(18)$ & $-0.0023(18)$ \\
Li2 & $0.012(2)$ & $0.014(2)$ & $0.013(2)$ & $-0.0009(17)$ & $0.0008(17)$ & $0.0009(17)$ \\
Li3 & $0.013(2)$ & $0.017(2)$ & $0.015(2)$ & $-0.0014(18)$ & $0.0000(18)$ & $-0.0016(18)$ \\
\hline
\end{tabular}


Geometric parameters $\left(\AA,{ }^{\circ}\right)$

\begin{tabular}{|c|c|c|c|}
\hline $\mathrm{Ga} 1-\mathrm{O} 1$ & $1.8385(18)$ & $\mathrm{B} 1-\mathrm{O} 2$ & $1.421(3)$ \\
\hline $\mathrm{Ga} 1-\mathrm{O} 2^{\mathrm{i}}$ & $1.8440(18)$ & $\mathrm{B} 1-\mathrm{O} 3$ & $1.339(3)$ \\
\hline $\mathrm{Ga} 1-\mathrm{O} 4$ & $1.8272(18)$ & $\mathrm{B} 2-\mathrm{O} 4$ & $1.393(3)$ \\
\hline $\mathrm{Ga} 1-\mathrm{O}^{\mathrm{ii}}$ & $1.8759(18)$ & $\mathrm{B} 2-\mathrm{O} 5$ & $1.423(3)$ \\
\hline $\mathrm{B} 1-\mathrm{O} 1$ & $1.393(3)$ & $\mathrm{B} 2-\mathrm{O} 6$ & $1.336(3)$ \\
\hline $\mathrm{O} 1-\mathrm{Ga} 1-\mathrm{O} 2^{\mathrm{i}}$ & $111.84(8)$ & Li1 $1^{\mathrm{xii}}-\mathrm{Li} 1-\mathrm{Li}^{\mathrm{vii}}$ & $149.2(3)$ \\
\hline $\mathrm{O} 1-\mathrm{Ga} 1-\mathrm{O} 5^{\mathrm{ii}}$ & $102.08(8)$ & $\mathrm{Li} 1^{\mathrm{xii}}-\mathrm{Li} 1-\mathrm{Li} 3^{\mathrm{ix}}$ & $72.2(2)$ \\
\hline $\mathrm{O} 1-\mathrm{Ga} 1-\mathrm{Li} 1^{\mathrm{iii}}$ & $116.15(10)$ & $\mathrm{Li} 2^{\mathrm{ix}}-\mathrm{Li} 1-\mathrm{Ga} 1^{\mathrm{iii}}$ & $63.94(14)$ \\
\hline $\mathrm{O} 1-\mathrm{Ga} 1-\mathrm{Li} 2^{\mathrm{iv}}$ & $124.51(10)$ & $\mathrm{Li} 2^{\mathrm{ix}}-\mathrm{Li} 1-\mathrm{Li} 3^{\mathrm{viii}}$ & $60.89(16)$ \\
\hline $\mathrm{O} 1-\mathrm{Ga} 1-\mathrm{Li}^{\mathrm{v}}$ & $35.98(10)$ & $\mathrm{Li} 2^{\mathrm{ix}}-\mathrm{Li} 1-\mathrm{Li}^{\mathrm{ii}}$ & $120.2(2)$ \\
\hline $\mathrm{O} 1-\mathrm{Ga} 1-\mathrm{Li} 2^{\mathrm{vi}}$ & $86.55(10)$ & $\mathrm{Li} 3^{\mathrm{ii}}-\mathrm{Li} 1-\mathrm{Ga} 1^{\mathrm{iii}}$ & $56.40(12)$ \\
\hline $\mathrm{O} 1-\mathrm{Ga} 1-\mathrm{Li} 2$ & $133.42(11)$ & $\mathrm{Li} 3^{\mathrm{vii}}-\mathrm{Li} 1-\mathrm{Ga} 1^{\mathrm{iii}}$ & $74.07(15)$ \\
\hline $\mathrm{O} 1-\mathrm{Ga} 1-\mathrm{Li} 3^{\mathrm{vii}}$ & $86.54(11)$ & Li $3^{\text {viii }-L i 1}-\mathrm{Ga} 1^{\mathrm{iii}}$ & $117.59(17)$ \\
\hline $\mathrm{O} 2^{\mathrm{i}}-\mathrm{Ga} 1-\mathrm{O} 5^{\mathrm{ii}}$ & $111.37(8)$ & $\mathrm{Li} 3^{\mathrm{ix}}-\mathrm{Li} 1-\mathrm{Ga} 1^{\mathrm{iii}}$ & $97.77(17)$ \\
\hline $\mathrm{O} 2^{\mathrm{i}}-\mathrm{Ga} 1-\mathrm{Li} 1^{\mathrm{iii}}$ & $76.62(10)$ & $\mathrm{Li}^{\mathrm{vii}}{ }^{\mathrm{vi}}-\mathrm{Li} 1-\mathrm{Li}^{\mathrm{ix}}$ & $117.0(2)$ \\
\hline $\mathrm{O} 2^{\mathrm{i}}-\mathrm{Ga} 1-\mathrm{Li}^{\mathrm{iv}}$ & $115.22(10)$ & $\mathrm{Li} 3^{\mathrm{ix}}-\mathrm{Li} 1-\mathrm{Li} 2^{\mathrm{ix}}$ & $114.0(2)$ \\
\hline $\mathrm{O} 2^{\mathrm{i}}-\mathrm{Ga} 1-\mathrm{Li}^{\mathrm{v}}$ & $135.30(11)$ & $\mathrm{Li} 3^{\mathrm{ii}}-\mathrm{Li} 1-\mathrm{Li} 3^{\mathrm{viii}}$ & $156.5(2)$ \\
\hline $\mathrm{O} 2^{\mathrm{i}}-\mathrm{Ga} 1-\mathrm{Li}^{\mathrm{vi}}$ & $37.59(10)$ & Li3 $3^{\text {vii }-L i 1}-\mathrm{Li}^{\text {viii }}$ & $107.55(19)$ \\
\hline $\mathrm{O} 2 \mathrm{i}-\mathrm{Ga} 1-\mathrm{Li} 2$ & $77.78(10)$ & $\mathrm{Li} 3^{\mathrm{ix}}-\mathrm{Li} 1-\mathrm{Li} 3^{\mathrm{viii}}$ & $129.60(18)$ \\
\hline $\mathrm{O} 2^{\mathrm{i}}-\mathrm{Ga} 1-\mathrm{Li} 3^{\mathrm{vii}}$ & $39.95(11)$ & $\mathrm{Li}^{\mathrm{vii}}-\mathrm{Li} 1-\mathrm{Li} 3^{\mathrm{ii}}$ & $49.58(19)$ \\
\hline $\mathrm{O} 4-\mathrm{Ga} 1-\mathrm{O} 1$ & $109.76(8)$ & $\mathrm{Li} 3^{\mathrm{ix}}-\mathrm{Li} 1-\mathrm{Li} 3^{\mathrm{ii}}$ & $73.05(19)$ \\
\hline $\mathrm{O} 4-\mathrm{Ga} 1-\mathrm{O} 2^{\mathrm{i}}$ & $110.94(8)$ & $\mathrm{Li} 3^{\mathrm{ix}}-\mathrm{Li} 1-\mathrm{Li} 3^{\mathrm{vii}}$ & $116.4(2)$ \\
\hline $\mathrm{O} 4-\mathrm{Ga} 1-\mathrm{O}^{\mathrm{ii}}$ & $110.54(8)$ & $\mathrm{Ga} 1-\mathrm{Li} 2-\mathrm{Ga}^{\mathrm{vi}}$ & $105.15(13)$ \\
\hline $\mathrm{O} 4-\mathrm{Ga} 1-\mathrm{Li} 1^{\mathrm{iii}}$ & $126.18(10)$ & $\mathrm{Ga} 1{ }^{\mathrm{i}}-\mathrm{Li} 2-\mathrm{Ga}^{\mathrm{iv}}$ & $70.59(10)$ \\
\hline $\mathrm{O} 4-\mathrm{Ga} 1-\mathrm{Li} 2$ & $34.37(10)$ & $\mathrm{Ga} 1-\mathrm{Li} 2-\mathrm{Ga}^{\mathrm{iv}}$ & $95.24(13)$ \\
\hline $\mathrm{O} 4-\mathrm{Ga} 1-\mathrm{Li} 2^{\mathrm{vi}}$ & $96.01(11)$ & $\mathrm{Ga} 1-\mathrm{Li} 2-\mathrm{Ga} 1$ & $104.73(14)$ \\
\hline $\mathrm{O} 4-\mathrm{Ga} 1-\mathrm{Li}^{\mathrm{v}}$ & $74.92(11)$ & 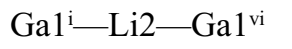 & $81.11(11)$ \\
\hline $\mathrm{O} 4-\mathrm{Ga} 1-\mathrm{Li} 2^{\mathrm{iv}}$ & $78.99(10)$ & $\mathrm{Ga} 1^{\mathrm{vi}}-\mathrm{Li} 2-\mathrm{Ga}^{\mathrm{iv}}$ & $148.57(16)$ \\
\hline $\mathrm{O} 4-\mathrm{Ga} 1-\mathrm{Li} 3^{\mathrm{vii}}$ & $150.82(11)$ & Ga1-Li2-Li3 ${ }^{\text {xiii }}$ & $135.80(19)$ \\
\hline $\mathrm{O} 5^{\mathrm{ii}}-\mathrm{Ga} 1-\mathrm{Li}^{\mathrm{iii}}$ & $34.75(10)$ & $\mathrm{Ga} 1^{\mathrm{i}}-\mathrm{Li} 2-\mathrm{Li} 3^{\mathrm{xiii}}$ & $110.84(17)$ \\
\hline $\mathrm{O} 5^{\mathrm{ii}}-\mathrm{Ga} 1-\mathrm{Li}^{2 \mathrm{iv}}$ & $33.37(10)$ & $\mathrm{B} 1^{\mathrm{viii}}-\mathrm{Li} 2-\mathrm{Ga}^{\mathrm{i}}$ & $136.51(18)$ \\
\hline $\mathrm{O}^{\mathrm{ii}}-\mathrm{Ga} 1-\mathrm{Li}^{\mathrm{v}}$ & $106.86(11)$ & 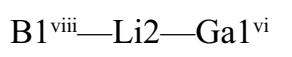 & $57.25(11)$ \\
\hline $\mathrm{O} 5^{\mathrm{ii}}-\mathrm{Ga} 1-\mathrm{Li}^{2 \mathrm{vi}}$ & $146.78(10)$ & $\mathrm{B} 1^{\mathrm{vii}}-\mathrm{Li} 2-\mathrm{Ga} 1^{\mathrm{iv}}$ & $152.84(18)$ \\
\hline $\mathrm{O} 5^{\mathrm{ii}}-\mathrm{Ga} 1-\mathrm{Li} 2$ & $116.90(11)$ & 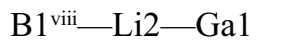 & $77.51(13)$ \\
\hline $\mathrm{O} 5^{\mathrm{ii}}-\mathrm{Ga} 1-\mathrm{Li}^{\mathrm{vii}}$ & $88.33(11)$ & $\mathrm{B} 1^{\mathrm{viii}}-\mathrm{Li} 2-\mathrm{B} 2^{\mathrm{i}}$ & $135.2(2)$ \\
\hline $\mathrm{Li} 1^{\mathrm{iii}}-\mathrm{Ga} 1-\mathrm{Li} 2^{\mathrm{iv}}$ & $52.55(11)$ & $\mathrm{B} 1^{\mathrm{vii}}-\mathrm{Li} 2-\mathrm{Li} 1^{\mathrm{xi}}$ & $97.11(18)$ \\
\hline $\mathrm{Li} 2^{\mathrm{v}}-\mathrm{Ga} 1-\mathrm{Li} 1^{\mathrm{iii}}$ & $137.36(13)$ & $\mathrm{B} 1^{\mathrm{viii}}-\mathrm{Li} 2-\mathrm{Li} 3^{\mathrm{xiii}}$ & $58.70(14)$ \\
\hline $\mathrm{Li} 2-\mathrm{Ga} 1-\mathrm{Li} 1^{\mathrm{iii}}$ & $110.42(13)$ & $\mathrm{B} 2 \mathrm{i}-\mathrm{Li} 2-\mathrm{Ga} 1$ & $146.69(19)$ \\
\hline $\mathrm{Li} 2^{\mathrm{vi}}-\mathrm{Ga} 1-\mathrm{Li} 1^{\mathrm{iii}}$ & $112.82(12)$ & $\mathrm{B} 2^{\mathrm{i}}-\mathrm{Li} 2-\mathrm{Ga}^{\mathrm{vi}}$ & $100.55(15)$ \\
\hline $\mathrm{Li} 2^{\mathrm{v}}-\mathrm{Ga} 1-\mathrm{Li} 2$ & $104.73(14)$ & $\mathrm{B} 2{ }^{\mathrm{i}}-\mathrm{Li} 2-\mathrm{Ga} 1^{\mathrm{i}}$ & $58.67(11)$ \\
\hline $\mathrm{Li} 2-\mathrm{Ga} 1-\mathrm{Li}^{\mathrm{vi}}$ & $74.85(13)$ & $\mathrm{B} 2^{\mathrm{i}}-\mathrm{Li} 2-\mathrm{Ga}^{\mathrm{iv}}$ & $53.11(10)$ \\
\hline $\mathrm{Li} 2^{\mathrm{vi}}-\mathrm{Ga} 1-\mathrm{Li} 2^{\mathrm{iv}}$ & $148.57(16)$ & 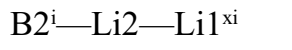 & $59.61(14)$ \\
\hline $\mathrm{Li} 2^{\mathrm{v}}-\mathrm{Ga} 1-\mathrm{Li} 2^{\mathrm{iv}}$ & $109.41(10)$ & $\mathrm{B} 2^{\mathrm{i}}-\mathrm{Li} 2-\mathrm{Li} 3^{\mathrm{xiii}}$ & $76.46(16)$ \\
\hline $\mathrm{Li} 2^{\mathrm{v}}-\mathrm{Ga} 1-\mathrm{Li}^{\mathrm{vi}}$ & $98.89(11)$ & $\mathrm{O} 1-\mathrm{i} i 2-\mathrm{Ga}^{\mathrm{vi}}$ & $72.19(15)$ \\
\hline $\mathrm{Li} 2-\mathrm{Ga} 1-\mathrm{Li}^{2}{ }^{\mathrm{iv}}$ & $84.76(13)$ & $\mathrm{O} 1$ - $-\mathrm{Li} 2-\mathrm{Ga} 1$ & $75.30(16)$ \\
\hline
\end{tabular}




\begin{tabular}{|c|c|c|c|}
\hline Li3 $3^{\mathrm{vii}}-\mathrm{Ga} 1-\mathrm{Li} 1^{\mathrm{iii}}$ & $59.79(12)$ & $\mathrm{O} 1{ }^{\mathrm{i}}-\mathrm{Li} 2-\mathrm{Ga} 1^{\mathrm{iv}}$ & $90.70(18)$ \\
\hline $\mathrm{Li}^{\mathrm{vii}}-\mathrm{Ga} 1-\mathrm{Li} 2^{\mathrm{vi}}$ & $59.91(13)$ & $\mathrm{O} 1-\mathrm{i} 2-\mathrm{Ga}^{\mathrm{i}}$ & $34.43(10)$ \\
\hline $\mathrm{Li} 3^{\mathrm{vii}}-\mathrm{Ga} 1-\mathrm{Li} 2^{\mathrm{v}}$ & $121.95(13)$ & $\mathrm{O} 1-\mathrm{i}$ i2-B1 $1^{\mathrm{viii}}$ & $112.1(2)$ \\
\hline $\mathrm{Li}^{\mathrm{vii}}-\mathrm{Ga} 1-\mathrm{Li} 2$ & $117.45(13)$ & $\mathrm{O} 1^{\mathrm{i}}-\mathrm{Li} 2-\mathrm{B} 2^{\mathrm{i}}$ & $93.10(19)$ \\
\hline $\mathrm{Li} 3^{\mathrm{vii}}-\mathrm{Ga} 1-\mathrm{Li} 2^{\mathrm{iv}}$ & $112.26(13)$ & $\mathrm{O} 1{ }^{\mathrm{i}}-\mathrm{Li} 2-\mathrm{O} 2^{\text {viii }}$ & $102.8(2)$ \\
\hline $\mathrm{O} 1-\mathrm{B} 1-\mathrm{O} 2$ & $115.8(2)$ & $\mathrm{O} 1^{\mathrm{i}}-\mathrm{Li} 2-\mathrm{O} 5^{\mathrm{i}}$ & $105.5(2)$ \\
\hline $\mathrm{O} 1-\mathrm{B} 1-\mathrm{Li} 2^{\text {viii }}$ & $104.8(2)$ & 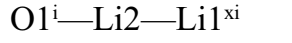 & $149.9(3)$ \\
\hline $\mathrm{O} 1-\mathrm{B} 1-\mathrm{Li} 3^{3 i}$ & $113.6(2)$ & $\mathrm{O} 1^{\mathrm{i}}-\mathrm{Li} 2-\mathrm{Li}^{\mathrm{xiii}}$ & $124.6(2)$ \\
\hline $\mathrm{O} 2-\mathrm{B} 1-\mathrm{Li} 2^{\mathrm{viii}}$ & $47.04(15)$ & $\mathrm{O} 2^{\mathrm{vii}}-\mathrm{Li} 2-\mathrm{Ga} 1^{\mathrm{vi}}$ & $32.82(9)$ \\
\hline $\mathrm{O} 2-\mathrm{B} 1-\mathrm{Li} 3^{\mathrm{ii}}$ & $111.9(2)$ & 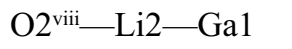 & $102.44(18)$ \\
\hline $\mathrm{O} 3-\mathrm{B} 1-\mathrm{O} 1$ & $123.3(2)$ & $\mathrm{O} 2^{\text {viii-Li2 } 2-\mathrm{Ga}^{\mathrm{iv}}}$ & $159.9(2)$ \\
\hline $\mathrm{O} 3-\mathrm{B} 1-\mathrm{O} 2$ & $120.9(2)$ & 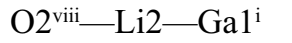 & $113.13(19)$ \\
\hline $\mathrm{O} 3-\mathrm{B} 1-\mathrm{Li} 2^{\mathrm{viii}}$ & $113.91(19)$ & $\mathrm{O} 2^{\mathrm{vii}}-\mathrm{Li} 2-\mathrm{B} 1^{\mathrm{viii}}$ & $30.07(10)$ \\
\hline $\mathrm{O} 3-\mathrm{B} 1-\mathrm{Li} 3^{\mathrm{ii}}$ & $42.00(16)$ & 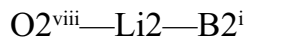 & $110.6(2)$ \\
\hline $\mathrm{Li} 3^{\mathrm{ii}}-\mathrm{B} 1-\mathrm{Li} 2^{\mathrm{viii}}$ & $141.61(18)$ & $\mathrm{O} 2^{\text {viii }-\mathrm{Li} 2-\mathrm{Li}^{\mathrm{xi}}}$ & $99.0(2)$ \\
\hline $\mathrm{O} 4-\mathrm{B} 2-\mathrm{O} 5$ & $117.1(2)$ & $\mathrm{O} 2^{\mathrm{vii}}-\mathrm{Li} 2-\mathrm{Li}^{\mathrm{xiii}}$ & $39.36(13)$ \\
\hline $\mathrm{O} 4-\mathrm{B} 2-\mathrm{Li} 1^{\mathrm{viii}}$ & $106.0(2)$ & $\mathrm{O} 4-\mathrm{Li} 2-\mathrm{Ga} 1$ & $33.11(10)$ \\
\hline $\mathrm{O} 4-\mathrm{B} 2-\mathrm{Li} 2^{\mathrm{v}}$ & $88.65(18)$ & $\mathrm{O} 4-\mathrm{Li} 2-\mathrm{Ga}^{\mathrm{vi}}$ & $121.9(2)$ \\
\hline $\mathrm{O} 4-\mathrm{B} 2-\mathrm{Li} 3$ & $149.2(2)$ & $\mathrm{O} 4-\mathrm{Li} 2-\mathrm{Ga}^{\mathrm{i}}$ & $131.9(2)$ \\
\hline $\mathrm{O} 5-\mathrm{B} 2-\mathrm{Li} 1^{\mathrm{viii}}$ & $119.8(2)$ & $\mathrm{O} 4-\mathrm{Li} 2-\mathrm{Ga}^{\mathrm{iv}}$ & $87.97(18)$ \\
\hline $\mathrm{O} 5-\mathrm{B} 2-\mathrm{Li} 2^{\mathrm{v}}$ & $41.16(15)$ & $\mathrm{O} 4-\mathrm{Li} 2-\mathrm{B} 1^{\mathrm{viii}}$ & $71.58(17)$ \\
\hline $\mathrm{O} 5-\mathrm{B} 2-\mathrm{Li} 3$ & $88.65(18)$ & $\mathrm{O} 4-\mathrm{Li} 2-\mathrm{B} 2^{\mathrm{i}}$ & $136.5(2)$ \\
\hline $\mathrm{O} 6-\mathrm{B} 2-\mathrm{O} 4$ & $121.2(2)$ & $\mathrm{O} 4-\mathrm{Li} 2-\mathrm{O} 1^{\mathrm{i}}$ & $107.7(2)$ \\
\hline $\mathrm{O} 6-\mathrm{B} 2-\mathrm{O} 5$ & $121.6(2)$ & $\mathrm{O} 4-\mathrm{Li} 2-\mathrm{O} 2^{\text {viii }}$ & $101.6(2)$ \\
\hline $\mathrm{O} 6-\mathrm{B} 2-\mathrm{Li1} 1^{\mathrm{viii}}$ & $40.79(15)$ & $\mathrm{O} 4-\mathrm{Li} 2-\mathrm{O} 5^{\mathrm{i}}$ & $108.5(2)$ \\
\hline $\mathrm{O} 6-\mathrm{B} 2-\mathrm{Li} 2^{\mathrm{v}}$ & $137.1(2)$ & $\mathrm{O} 4-\mathrm{Li} 2-\mathrm{Li}^{\mathrm{xi}}$ & $87.7(2)$ \\
\hline $\mathrm{O} 6-\mathrm{B} 2-\mathrm{Li} 3$ & $37.74(15)$ & $\mathrm{O} 4-\mathrm{Li} 2-\mathrm{Li} 3^{\mathrm{xiii}}$ & $117.1(2)$ \\
\hline $\mathrm{Li}^{\mathrm{viii}}-\mathrm{B} 2-\mathrm{Li} 2^{\mathrm{v}}$ & $105.56(17)$ & $\mathrm{O} 5-\mathrm{Li} 2-\mathrm{Ga}^{\mathrm{iv}}$ & $31.06(10)$ \\
\hline $\mathrm{Li}^{\mathrm{viii}}-\mathrm{B} 2-\mathrm{Li} 3$ & $70.99(16)$ & $\mathrm{O} 5-\mathrm{Li} 2-\mathrm{Ga}^{\mathrm{i}}$ & $74.16(15)$ \\
\hline $\mathrm{Li} 3-\mathrm{B} 2-\mathrm{Li} 2^{\mathrm{v}}$ & $122.03(17)$ & $\mathrm{O} 5-\mathrm{Li} 2-\mathrm{Ga}^{\mathrm{i}}{ }^{\mathrm{vi}}$ & $127.9(2)$ \\
\hline $\mathrm{Ga} 1-\mathrm{O} 1-\mathrm{Li}^{\mathrm{v}}$ & $109.59(17)$ & $\mathrm{O} 5-\mathrm{Li} 2-\mathrm{Ga} 1$ & $125.0(2)$ \\
\hline $\mathrm{B} 1-\mathrm{O} 1-\mathrm{Ga} 1$ & $120.01(17)$ & $\mathrm{O} 5^{\mathrm{i}}-\mathrm{Li} 2-\mathrm{B} 1^{\mathrm{viii}}$ & $140.4(2)$ \\
\hline $\mathrm{B} 1-\mathrm{O} 1-\mathrm{Li}^{\mathrm{v}}$ & $129.9(2)$ & $\mathrm{O} 5^{\mathrm{i}}-\mathrm{Li} 2-\mathrm{B} 2^{\mathrm{i}}$ & $27.93(10)$ \\
\hline $\mathrm{Ga}^{\mathrm{v}}-\mathrm{O} 2-\mathrm{Li}^{\mathrm{viii}}$ & $109.59(15)$ & $\mathrm{O} 5-\mathrm{i} 2-\mathrm{O} 2^{\mathrm{viii}}$ & $129.2(3)$ \\
\hline $\mathrm{Ga}^{\mathrm{v}}-\mathrm{O} 2-\mathrm{Li}^{\mathrm{ix}}$ & $103.87(16)$ & $\mathrm{O} 5^{\mathrm{i}}-\mathrm{Li} 2-\mathrm{Li}^{\mathrm{xi}}$ & $44.44(15)$ \\
\hline $\mathrm{B} 1-\mathrm{O} 2-\mathrm{Ga}^{\mathrm{v}}$ & $123.74(16)$ & $\mathrm{O} 5^{\mathrm{i}}-\mathrm{Li} 2-\mathrm{Li}^{\mathrm{xiii}}$ & $90.06(19)$ \\
\hline $\mathrm{B} 1-\mathrm{O} 2-\mathrm{Li} 2^{\mathrm{viii}}$ & $102.9(2)$ & $\mathrm{Li}^{\mathrm{xi}}-\mathrm{Li} 2-\mathrm{Ga}^{\mathrm{vi}}$ & $121.89(19)$ \\
\hline $\mathrm{B} 1-\mathrm{O} 2-\mathrm{Li}^{\mathrm{ix}}$ & $114.5(2)$ & $\mathrm{Li} 1^{\mathrm{xi}}-\mathrm{Li} 2-\mathrm{Ga}^{\mathrm{i}}$ & $116.94(18)$ \\
\hline $\mathrm{Li}^{\mathrm{ix}}-\mathrm{O} 2-\mathrm{Li} 2^{\text {viii }}$ & $99.6(2)$ & 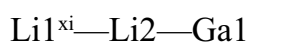 & $119.78(18)$ \\
\hline $\mathrm{B} 1-\mathrm{O} 3-\mathrm{Li} 1$ & $120.6(2)$ & $\mathrm{Li}^{\mathrm{xi}}-\mathrm{Li} 2-\mathrm{Ga}^{\mathrm{iv}}$ & $63.51(13)$ \\
\hline $\mathrm{B} 1-\mathrm{O} 3-\mathrm{Li}^{\mathrm{vii}}$ & $133.1(2)$ & $\mathrm{Li}^{\mathrm{xi}}-\mathrm{Li} 2-\mathrm{Li} 3^{\mathrm{xiii}}$ & $64.81(16)$ \\
\hline $\mathrm{B} 1-\mathrm{O} 3-\mathrm{Li} 3^{\mathrm{ii}}$ & $110.7(2)$ & $\mathrm{Li} 3^{\text {xiii }}-\mathrm{Li} 2-\mathrm{Ga} 1^{\mathrm{iv}}$ & $120.57(17)$ \\
\hline $\mathrm{Li} 1-\mathrm{O} 3-\mathrm{Li} 3^{\mathrm{ii}}$ & $108.2(2)$ & $\mathrm{Li} 3^{\mathrm{xiii}}-\mathrm{Li} 2-\mathrm{Ga}^{\mathrm{vi}}$ & $57.29(12)$ \\
\hline $\mathrm{Li} 1-\mathrm{O} 3-\mathrm{Li}^{\text {vii }}$ & $95.7(2)$ & $\mathrm{Ga}^{\mathrm{x}}-\mathrm{Li} 3-\mathrm{Li} 1^{\mathrm{ii}}$ & $63.82(13)$ \\
\hline $\mathrm{Li}^{\mathrm{vii}}-\mathrm{O} 3-\mathrm{Li}^{\mathrm{ii}}$ & $81.0(2)$ & $\mathrm{Ga} 1^{\mathrm{x}}-\mathrm{Li} 3-\mathrm{Li}^{\mathrm{xiii}}$ & $62.79(13)$ \\
\hline $\mathrm{Ga} 1-\mathrm{O} 4-\mathrm{Li} 2$ & $112.52(17)$ & 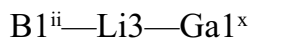 & $121.35(18)$ \\
\hline $\mathrm{B} 2-\mathrm{O} 4-\mathrm{Ga} 1$ & $127.86(17)$ & 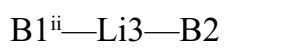 & $69.64(14)$ \\
\hline $\mathrm{B} 2-\mathrm{O} 4-\mathrm{Li} 2$ & $119.6(2)$ & $\mathrm{B} 1^{\mathrm{ii}}-\mathrm{Li} 3-\mathrm{Li} 1^{\mathrm{ii}}$ & $57.56(14)$ \\
\hline
\end{tabular}




\begin{tabular}{|c|c|c|c|}
\hline $\mathrm{Ga}^{\mathrm{ii}}-\mathrm{O} 5-\mathrm{Li}^{\mathrm{x}}$ & $113.47(16)$ & $\mathrm{B} 1^{\mathrm{ii}}-\mathrm{Li} 3-\mathrm{Li}^{\mathrm{x}}$ & $106.5(2)$ \\
\hline $\mathrm{Ga} 1^{\mathrm{ii}}-\mathrm{O} 5-\mathrm{Li} 2^{\mathrm{v}}$ & $115.56(17)$ & $\mathrm{B} 1^{\mathrm{ii}}-\mathrm{Li} 3-\mathrm{Li} 1^{\mathrm{xi}}$ & $80.20(17)$ \\
\hline $\mathrm{B} 2-\mathrm{O} 5-\mathrm{Ga}^{\mathrm{ii}}$ & $113.05(16)$ & $\mathrm{B} 1^{\mathrm{ii}}-\mathrm{Li} 3-\mathrm{Li} 2^{\mathrm{xiii}}$ & $150.9(2)$ \\
\hline $\mathrm{B} 2-\mathrm{O} 5-\mathrm{Li} 1^{\mathrm{x}}$ & $110.1(2)$ & $\mathrm{B} 2-\mathrm{Li} 3-\mathrm{Ga}^{\mathrm{x}}$ & $168.9(2)$ \\
\hline $\mathrm{B} 2-\mathrm{O} 5-\mathrm{Li} 2^{\mathrm{v}}$ & $110.9(2)$ & $\mathrm{B} 2-\mathrm{Li} 3-\mathrm{Li} 1^{\mathrm{x}}$ & $60.43(15)$ \\
\hline $\mathrm{Li}^{2}-\mathrm{O} 5-\mathrm{Li}^{\mathrm{x}}$ & $92.0(2)$ & $\mathrm{B} 2-\mathrm{Li} 3-\mathrm{Li} 1^{\mathrm{ii}}$ & $127.1(2)$ \\
\hline $\mathrm{B} 2-\mathrm{O} 6-\mathrm{Li} 1^{\text {viii }}$ & $112.8(2)$ & $\mathrm{B} 2-\mathrm{Li} 3-\mathrm{Li}^{\mathrm{xi}}$ & $64.26(15)$ \\
\hline $\mathrm{B} 2-\mathrm{O} 6-\mathrm{Li}^{\mathrm{xi}}$ & $130.9(2)$ & $\mathrm{B} 2-\mathrm{Li} 3-\mathrm{Li} 2^{\mathrm{xiii}}$ & $108.51(19)$ \\
\hline $\mathrm{B} 2-\mathrm{O} 6-\mathrm{Li} 3$ & $117.0(2)$ & $\mathrm{O} 2^{\mathrm{xi}}-\mathrm{Li} 3-\mathrm{Ga}^{\mathrm{x}}$ & $36.18(10)$ \\
\hline $\mathrm{Li} 1^{\mathrm{xi}}-\mathrm{O} 6-\mathrm{Li1}{ }^{\mathrm{vii}}$ & $83.6(2)$ & $\mathrm{O} 2^{\mathrm{xi}}-\mathrm{Li} 3-\mathrm{B} 1^{\mathrm{ii}}$ & $122.9(2)$ \\
\hline $\mathrm{Li} 3-\mathrm{O} 6-\mathrm{Li} 1^{\mathrm{xi}}$ & $95.2(2)$ & $\mathrm{O} 2{ }^{\mathrm{xi}}-\mathrm{Li} 3-\mathrm{B} 2$ & $141.0(2)$ \\
\hline Li3-O6-Li1 $1^{\text {viii }}$ & $112.6(2)$ & $\mathrm{O} 2^{\mathrm{xi}}-\mathrm{Li} 3-\mathrm{Li} 1^{\mathrm{ii}}$ & $77.94(18)$ \\
\hline $\mathrm{B} 2^{\mathrm{vii}}-\mathrm{Li} 1-\mathrm{Ga} 1^{\mathrm{iii}}$ & $150.65(19)$ & $\mathrm{O} 2^{\mathrm{xi}}-\mathrm{Li} 3-\mathrm{Li}^{\mathrm{x}}$ & $130.1(2)$ \\
\hline $\mathrm{B} 2^{\mathrm{viii}}-\mathrm{Li} 1-\mathrm{Li} 2^{\mathrm{ix}}$ & $115.6(2)$ & $\mathrm{O} 2^{\mathrm{xi}}-\mathrm{Li} 3-\mathrm{Li} 1^{\mathrm{xi}}$ & $80.86(19)$ \\
\hline $\mathrm{B} 2^{\text {viii }-\mathrm{Li} 1-\mathrm{Li} 3^{\mathrm{ix}}}$ & $107.8(2)$ & $\mathrm{O} 2^{\mathrm{xi}}-\mathrm{Li} 3-\mathrm{Li}^{2 \mathrm{xii}}$ & $41.01(14)$ \\
\hline $\mathrm{B} 2^{\mathrm{viii}}-\mathrm{Li} 1-\mathrm{Li} 3^{\mathrm{ii}}$ & $117.46(19)$ & $\mathrm{O} 2^{\mathrm{xi}}-\mathrm{Li} 3-\mathrm{Li}^{3 \mathrm{xiv}}$ & $118.1(3)$ \\
\hline $\mathrm{B} 2^{\mathrm{viii}}-\mathrm{Li} 1-\mathrm{Li} 3^{\mathrm{vii}}$ & $81.65(18)$ & $\mathrm{O} 3^{\mathrm{ii}}-\mathrm{Li} 3-\mathrm{Ga}^{\mathrm{x}}$ & $97.47(18)$ \\
\hline 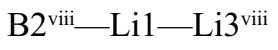 & $54.76(14)$ & $\mathrm{O} 3^{\mathrm{x}}-\mathrm{Li} 3-\mathrm{Ga}^{\mathrm{x}}$ & $73.34(15)$ \\
\hline $\mathrm{O} 3-\mathrm{Li} 1-\mathrm{Ga} 1^{\mathrm{iii}}$ & $90.74(18)$ & $\mathrm{O} 3^{\mathrm{x}}-\mathrm{Li} 3-\mathrm{B} 1^{\mathrm{ii}}$ & $116.5(2)$ \\
\hline $\mathrm{O} 3-\mathrm{Li1}-\mathrm{B} 2^{\mathrm{viii}}$ & $81.99(18)$ & $\mathrm{O} 3^{\mathrm{ii}}-\mathrm{Li} 3-\mathrm{B} 1^{\mathrm{ii}}$ & $27.27(10)$ \\
\hline $\mathrm{O} 3-\mathrm{Li} 1-\mathrm{O} 5^{\mathrm{vii}}$ & $110.1(2)$ & $\mathrm{O} 3^{x}-\mathrm{Li} 3-\mathrm{B} 2$ & $101.3(2)$ \\
\hline $\mathrm{O} 3-\mathrm{Li} 1-\mathrm{O}^{\mathrm{viii}}$ & $108.0(2)$ & 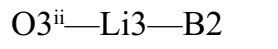 & $92.9(2)$ \\
\hline O3-Li1-O6 $6^{\mathrm{ix}}$ & $117.7(3)$ & $\mathrm{O} 3^{x}-\mathrm{Li} 3-\mathrm{O} 2^{\mathrm{xi}}$ & $103.3(2)$ \\
\hline O3-Li1-Li1 ${ }^{x i i}$ & $125.5(3)$ & $\mathrm{O} 3^{\mathrm{ii}}-\mathrm{Li} 3-\mathrm{O} 2^{\mathrm{xi}}$ & $112.5(2)$ \\
\hline $\mathrm{O} 3-\mathrm{Li} 1-\mathrm{Li} 2^{\mathrm{ix}}$ & $153.3(3)$ & $\mathrm{O} 3^{\mathrm{x}}-\mathrm{Li} 3-\mathrm{O} 3^{\mathrm{ii}}$ & $99.0(2)$ \\
\hline $\mathrm{O} 3-\mathrm{Li} 1-\mathrm{Li} 3^{\mathrm{vii}}$ & $42.42(15)$ & $\mathrm{O}^{\mathrm{ii}}-\mathrm{Li} 3-\mathrm{Li1}^{\mathrm{x}}$ & $108.3(2)$ \\
\hline $\mathrm{O} 3-\mathrm{Li} 1-\mathrm{Li} 3^{\mathrm{ii}}$ & $36.20(14)$ & $\mathrm{O} 3^{\mathrm{ii}}-\mathrm{Li} 3-\mathrm{Li} 1^{\mathrm{xi}}$ & $103.2(2)$ \\
\hline $\mathrm{O} 3$ - Li1-Li3 ${ }^{\text {viii }}$ & $133.3(2)$ & O3 ${ }^{x}-\mathrm{Li} 3-\mathrm{Li}^{\mathrm{x}}$ & $41.91(14)$ \\
\hline $\mathrm{O} 3-\mathrm{Li} 1-\mathrm{Li} 3^{\mathrm{ix}}$ & $75.85(19)$ & $\mathrm{O} 3^{\mathrm{ii}}-\mathrm{Li} 3-\mathrm{Li}^{\mathrm{ii}}$ & $35.65(13)$ \\
\hline $\mathrm{O} 5^{\mathrm{vii}}-\mathrm{Li} 1-\mathrm{Ga}^{\mathrm{iii}}$ & $31.78(9)$ & $\mathrm{O} 3^{\mathrm{x}}-\mathrm{Li} 3-\mathrm{Li} 1^{\mathrm{ii}}$ & $99.1(2)$ \\
\hline $\mathrm{O} 5^{\mathrm{vii}}-\mathrm{Li} 1-\mathrm{B} 2^{\mathrm{viii}}$ & $126.2(2)$ & $\mathrm{O} 3^{\mathrm{x}}-\mathrm{Li} 3-\mathrm{Li}^{\mathrm{xi}}$ & $153.9(3)$ \\
\hline O5 ${ }^{\mathrm{vii}}-\mathrm{Li} 1-\mathrm{Li1}^{\mathrm{xii}}$ & $124.5(3)$ & $\mathrm{O} 3^{\mathrm{ii}}-\mathrm{Li} 3-\mathrm{Li} 2^{\mathrm{xiii}}$ & $153.3(2)$ \\
\hline $\mathrm{O}^{\mathrm{vii}}-\mathrm{Li} 1-\mathrm{Li}^{\mathrm{ix}}$ & $43.60(14)$ & $\mathrm{O} 3^{\mathrm{x}}-\mathrm{Li} 3-\mathrm{Li}^{\mathrm{xiii}}$ & $92.5(2)$ \\
\hline $\mathrm{O} 5^{\mathrm{vii}}-\mathrm{Li} 1-\mathrm{Li}^{\text {viii }}$ & $86.42(18)$ & $\mathrm{O} 3^{\mathrm{ii}}-\mathrm{Li} 3-\mathrm{Li} 3^{\mathrm{xiv}}$ & $49.37(18)$ \\
\hline $\mathrm{O} 5^{\mathrm{vii}-\mathrm{Li} 1-\mathrm{Li} 3^{\mathrm{ix}}}$ & $126.0(2)$ & $\mathrm{O}^{\mathrm{x}}-\mathrm{Li} 3-\mathrm{Li} 3^{\mathrm{xiv}}$ & $49.60(17)$ \\
\hline $\mathrm{O}^{\mathrm{vii}}-\mathrm{Li} 1-\mathrm{Li} 3^{\mathrm{ii}}$ & $82.63(18)$ & $\mathrm{O} 6-\mathrm{Li} 3-\mathrm{Ga}^{\mathrm{x}}$ & $149.2(2)$ \\
\hline $\mathrm{O}^{\mathrm{vii}}-\mathrm{Li} 1-\mathrm{Li}^{\mathrm{vii}}$ & $76.30(19)$ & $\mathrm{O} 6-\mathrm{Li} 3-\mathrm{B} 1^{\mathrm{ii}}$ & $82.26(18)$ \\
\hline $\mathrm{O} 6^{\mathrm{ix}}-\mathrm{Li} 1-\mathrm{Ga}^{\mathrm{iii}}$ & $95.39(18)$ & $\mathrm{O} 6-\mathrm{Li} 3-\mathrm{B} 2$ & $25.26(10)$ \\
\hline $\mathrm{O}^{\mathrm{vii}}-\mathrm{Li} 1-\mathrm{Ga}^{\mathrm{iii}}$ & $149.8(2)$ & $\mathrm{O} 6-\mathrm{Li} 3-\mathrm{O} 2^{\mathrm{xi}}$ & $115.8(3)$ \\
\hline 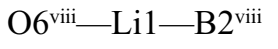 & $26.37(10)$ & $\mathrm{O} 6-\mathrm{Li} 3-\mathrm{O}^{\mathrm{ii}}$ & $109.1(2)$ \\
\hline $\mathrm{O}^{\mathrm{ix}}-\mathrm{Li} 1-\mathrm{B} 2^{\mathrm{viii}}$ & $113.2(2)$ & $\mathrm{O} 6-\mathrm{Li} 3-\mathrm{O}^{\mathrm{x}}$ & $116.1(3)$ \\
\hline $\mathrm{O} 6^{\mathrm{vii}}-\mathrm{Li} 1-\mathrm{O} 5^{\mathrm{vii}}$ & $118.0(2)$ & O6- $-\mathrm{Li} 3-\mathrm{Li}^{\mathrm{x}}$ & $74.64(19)$ \\
\hline $\mathrm{O}^{\mathrm{ix}}-\mathrm{Li} 1-\mathrm{O} 5^{\mathrm{vii}}$ & $106.5(2)$ & $\mathrm{O} 6-\mathrm{Li} 3-\mathrm{Li} 1^{\mathrm{xi}}$ & $42.94(15)$ \\
\hline $\mathrm{O}^{\mathrm{ix}}-\mathrm{Li} 1-\mathrm{O}^{\mathrm{viii}}$ & $96.4(2)$ & $\mathrm{O} 6-\mathrm{Li} 3-\mathrm{Li} 1^{\mathrm{ii}}$ & $135.9(2)$ \\
\hline O6 ${ }^{\text {viii }}-\mathrm{Li} 1-\mathrm{Li} 1^{\mathrm{xii}}$ & $48.01(17)$ & $\mathrm{O} 6-\mathrm{Li} 3-\mathrm{Li} 2^{\mathrm{xiii}}$ & $86.9(2)$ \\
\hline $\mathrm{O} 6^{\mathrm{ix}}-\mathrm{Li} 1-\mathrm{Li1} 1^{\mathrm{xii}}$ & $48.35(17)$ & $\mathrm{O} 6-\mathrm{Li} 3-\mathrm{Li} 3^{\mathrm{xiv}}$ & $126.2(3)$ \\
\hline O6 ${ }^{\text {viii }}-\mathrm{Li} 1-\mathrm{Li} 2^{\mathrm{ix}}$ & $92.4(2)$ & $\mathrm{Li}^{\mathrm{x}}-\mathrm{Li} 3-\mathrm{Ga}^{\mathrm{x}}$ & $112.15(18)$ \\
\hline $\mathrm{O}^{\mathrm{ix}}-\mathrm{Li} 1-\mathrm{Li} 2^{\mathrm{ix}}$ & $75.35(18)$ & $\mathrm{Li}^{\mathrm{xi}}-\mathrm{Li} 3-\mathrm{Ga}^{\mathrm{x}}$ & $116.62(19)$ \\
\hline
\end{tabular}




\begin{tabular}{|c|c|c|c|}
\hline $\mathrm{O} 6^{\mathrm{vii}}-\mathrm{Li} 1-\mathrm{Li} 3^{\mathrm{vii}}$ & $103.7(2)$ & $\mathrm{Li}^{\mathrm{x}}-\mathrm{Li} 3-\mathrm{Li}^{\mathrm{ii}}$ & 130.42 (19) \\
\hline $\mathrm{O}^{\mathrm{ix}}-\mathrm{Li} 1-\mathrm{Li}^{3 i}$ & $106.1(2)$ & $\mathrm{Li}^{\mathrm{xi}}-\mathrm{Li} 3-\mathrm{Li}^{\mathrm{x}}$ & $116.4(2)$ \\
\hline O6 $6^{\text {viii }-L i 1-L i 3 ~} 3^{\text {ii }}$ & $143.8(2)$ & $\mathrm{Li}^{\mathrm{xi}}-\mathrm{Li} 3-\mathrm{Li}^{\mathrm{ii}}$ & $106.95(18)$ \\
\hline $\mathrm{O} 6^{\mathrm{ix}}-\mathrm{Li} 1-\mathrm{Li}^{\mathrm{vii}}$ & $155.5(3)$ & $\operatorname{Li} 1^{x}-\operatorname{Li} 3-\operatorname{Li} 2^{x i i i}$ & $96.3(2)$ \\
\hline O6 $6^{\text {viii_-Li1 }-\mathrm{Li}^{\text {ix }}}$ & $109.5(2)$ & $\mathrm{Li}^{\mathrm{xi}}-\mathrm{Li} 3-\mathrm{Li}^{\mathrm{xii}}$ & $73.57(18)$ \\
\hline $\mathrm{O} 6^{\mathrm{ix}}-\mathrm{Li} 1-\mathrm{Li} 3^{\mathrm{ix}}$ & $41.90(15)$ & $\mathrm{Li} 2^{\mathrm{xiii}}-\mathrm{Li} 3-\mathrm{Li}^{\mathrm{ii}}$ & $118.79(19)$ \\
\hline $\mathrm{O} 6^{\mathrm{ix}}-\mathrm{Li} 1-\mathrm{Li}^{\mathrm{viii}}$ & $96.9(2)$ & $\mathrm{Li} 3^{\mathrm{xiv}}-\mathrm{Li} 3-\mathrm{Ga}^{\mathrm{x}}$ & $83.1(2)$ \\
\hline $\mathrm{O}^{\mathrm{viii}}-\mathrm{Li} 1-\mathrm{Li} 3^{\mathrm{viii}}$ & $33.21(13)$ & $\mathrm{Li}^{\mathrm{xiv}}-\mathrm{Li} 3-\mathrm{B} 1^{\mathrm{ii}}$ & $70.0(2)$ \\
\hline Li1 ${ }^{\mathrm{xii}}-\mathrm{Li} 1-\mathrm{Ga} 1^{\mathrm{iii}}$ & $136.1(3)$ & $\mathrm{Li} 3^{\mathrm{xiv}}-\mathrm{Li} 3-\mathrm{B} 2$ & $100.9(3)$ \\
\hline 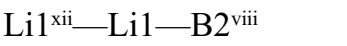 & $67.7(2)$ & $\mathrm{Li}^{\mathrm{xiv}}-\mathrm{Li} 3-\mathrm{Li}^{\mathrm{xi}}$ & $150.0(3)$ \\
\hline Li1 $1^{\mathrm{xii}}-\mathrm{Li} 1-\mathrm{Li} 2^{\mathrm{ix}}$ & $81.0(2)$ & $\mathrm{Li}^{\mathrm{xiv}}-\mathrm{Li} 3-\mathrm{Li}^{\mathrm{ii}}$ & $59.6(2)$ \\
\hline Li1 $1^{\text {xii }}-\mathrm{Li} 1-\mathrm{Li} 3^{\mathrm{viii}}$ & $57.4(2)$ & $\mathrm{Li}^{\mathrm{xiv}}-\mathrm{Li} 3-\mathrm{Li}^{\mathrm{x}}$ & $70.8(2)$ \\
\hline $\mathrm{Li}^{\mathrm{xii}}-\mathrm{Li} 1-\mathrm{Li} 3^{\mathrm{ii}}$ & $144.5(3)$ & $\mathrm{Li}^{\mathrm{xiv}}-\mathrm{Li} 3-\mathrm{Li}^{\mathrm{xiii}}$ & $136.2(3)$ \\
\hline $\mathrm{Ga} 1-\mathrm{O} 4-\mathrm{Li} 2-\mathrm{Ga}^{\mathrm{iv}}$ & $-102.80(13)$ & $\mathrm{O} 6-\mathrm{B} 2-\mathrm{O} 4-\mathrm{Li} 2$ & $0.4(4)$ \\
\hline $\mathrm{Ga} 1-\mathrm{O} 4-\mathrm{Li} 2-\mathrm{Ga}^{\mathrm{vi}}$ & $66.9(3)$ & $\mathrm{O} 6-\mathrm{B} 2-\mathrm{O} 5-\mathrm{Ga}^{\mathrm{ii}}$ & $-101.3(3)$ \\
\hline $\mathrm{Ga} 1-\mathrm{O} 4-\mathrm{Li} 2-\mathrm{Ga}^{\mathrm{i}}$ & $-41.4(3)$ & $\mathrm{O} 6-\mathrm{B} 2-\mathrm{O} 5-\mathrm{Li}^{\mathrm{x}}$ & $26.8(3)$ \\
\hline $\mathrm{Ga} 1-\mathrm{O} 4-\mathrm{Li} 2-\mathrm{B} 1^{\text {vii }}$ & $95.37(14)$ & $\mathrm{O} 6-\mathrm{B} 2-\mathrm{O} 5-\mathrm{Li}^{\mathrm{v}}$ & $127.1(3)$ \\
\hline $\mathrm{Ga} 1-\mathrm{O} 4-\mathrm{Li} 2-\mathrm{B} 2^{\mathrm{i}}$ & $-127.4(3)$ & $\mathrm{Li} 1 \mathrm{ii}-\mathrm{Ga} 1-\mathrm{O} 1-\mathrm{B} 1$ & $-37.0(2)$ \\
\hline $\mathrm{Ga} 1-\mathrm{O} 4-\mathrm{Li} 2-\mathrm{O}^{\mathrm{i}}$ & $-12.7(3)$ & $\mathrm{Li}^{\mathrm{iii}}-\mathrm{Ga} 1-\mathrm{O} 1-\mathrm{Li} 2^{\mathrm{v}}$ & $135.94(19)$ \\
\hline $\mathrm{Ga} 1-\mathrm{O} 4-\mathrm{Li} 2-\mathrm{O} 2^{\mathrm{viii}}$ & $95.0(2)$ & $\mathrm{Li} 1{ }^{\mathrm{iii}}-\mathrm{Ga} 1-\mathrm{O} 4-\mathrm{B} 2$ & $-105.3(2)$ \\
\hline $\mathrm{Ga} 1-\mathrm{O} 4-\mathrm{Li} 2-\mathrm{O}^{\mathrm{i}}$ & $-126.46(19)$ & $\mathrm{Li} 1^{\mathrm{iii}}-\mathrm{Ga} 1-\mathrm{O} 4-\mathrm{Li} 2$ & $72.3(2)$ \\
\hline $\mathrm{Ga} 1-\mathrm{O} 4-\mathrm{Li} 2-\mathrm{Li}^{\mathrm{xi}}$ & $-166.36(14)$ & $\mathrm{Li}^{\text {viii }}-\mathrm{B} 2-\mathrm{O} 4-\mathrm{Ga} 1$ & $-140.43(17)$ \\
\hline $\mathrm{Ga} 1-\mathrm{O} 4-\mathrm{Li} 2-\mathrm{Li}^{\mathrm{xii}}$ & $133.58(18)$ & $\mathrm{Li} 1{ }^{\text {viii- }} \mathrm{B} 2-\mathrm{O} 4-\mathrm{Li} 2$ & $42.1(3)$ \\
\hline $\mathrm{B} 2-\mathrm{O} 4-\mathrm{Li} 2-\mathrm{Ga}^{\mathrm{iv}}$ & $75.0(2)$ & $\mathrm{Li}^{\mathrm{viii}}-\mathrm{B} 2-\mathrm{O} 5-\mathrm{Ga} 1^{\mathrm{ii}}$ & $-149.04(15)$ \\
\hline $\mathrm{B} 2-\mathrm{O} 4-\mathrm{Li} 2-\mathrm{Ga}^{\mathrm{vi}}$ & $-115.2(2)$ & $\mathrm{Li} 1^{\mathrm{vii}}-\mathrm{B} 2-\mathrm{O} 5-\mathrm{Li}^{\mathrm{x}}$ & $-21.0(3)$ \\
\hline $\mathrm{B} 2-\mathrm{O} 4-\mathrm{Li} 2-\mathrm{Ga}^{\mathrm{i}}$ & $136.4(2)$ & $\mathrm{Li} 1^{\mathrm{vii}}-\mathrm{B} 2-\mathrm{O} 5-\mathrm{Li} 2^{\mathrm{v}}$ & $79.3(3)$ \\
\hline $\mathrm{B} 2-\mathrm{O} 4-\mathrm{Li} 2-\mathrm{Ga} 1$ & $177.8(3)$ & $\mathrm{Li1}^{\mathrm{vii}}-\mathrm{B} 2-\mathrm{O} 6-\mathrm{Li} 1^{\mathrm{xi}}$ & $-101.9(3)$ \\
\hline $\mathrm{B} 2-\mathrm{O} 4-\mathrm{Li} 2-\mathrm{B} 1^{\mathrm{viii}}$ & $-86.8(2)$ & $\mathrm{Li} 11^{\mathrm{viii}}-\mathrm{B} 2-\mathrm{O} 6-\mathrm{Li} 3$ & $133.1(3)$ \\
\hline $\mathrm{B} 2-\mathrm{O} 4-\mathrm{Li} 2-\mathrm{B} 2^{\mathrm{i}}$ & $50.5(4)$ & $\mathrm{Li} 2^{\mathrm{vi}}-\mathrm{Ga} 1-\mathrm{O} 1-\mathrm{B} 1$ & $76.7(2)$ \\
\hline $\mathrm{B} 2-\mathrm{O} 4-\mathrm{Li} 2-\mathrm{O}^{\mathrm{i}}$ & $165.1(2)$ & $\mathrm{Li} 2 \mathrm{v}-\mathrm{Ga} 1-\mathrm{O} 1-\mathrm{B} 1$ & $-173.0(3)$ \\
\hline $\mathrm{B} 2-\mathrm{O} 4-\mathrm{Li} 2-\mathrm{O} 2^{\text {viii }}$ & $-87.2(3)$ & $\mathrm{Li} 2^{\mathrm{iv}}-\mathrm{Ga} 1-\mathrm{O} 1-\mathrm{B} 1$ & $-98.1(2)$ \\
\hline $\mathrm{B} 2-\mathrm{O} 4-\mathrm{Li} 2-\mathrm{O}^{\mathrm{i}}$ & $51.4(3)$ & $\mathrm{Li} 2-\mathrm{Ga} 1-\mathrm{O} 1-\mathrm{B} 1$ & $142.1(2)$ \\
\hline $\mathrm{B} 2-\mathrm{O} 4-\mathrm{Li} 2-\mathrm{Li}^{\mathrm{xi}}$ & $11.5(3)$ & $\mathrm{Li} 2-\mathrm{Ga} 1-\mathrm{O} 1-\mathrm{Li}^{\mathrm{v}}$ & $-44.96(17)$ \\
\hline $\mathrm{B} 2-\mathrm{O} 4-\mathrm{Li} 2-\mathrm{Li}^{\mathrm{xiii}}$ & $-48.6(3)$ & $\mathrm{Li} 2^{\mathrm{vi}}-\mathrm{Ga} 1-\mathrm{O} 1-\mathrm{Li}^{\mathrm{v}}$ & $-110.28(16)$ \\
\hline $\mathrm{O} 1-\mathrm{Ga} 1-\mathrm{O} 4-\mathrm{B} 2$ & $42.2(2)$ & $\mathrm{Li} 2^{\mathrm{iv}}-\mathrm{Ga} 1-\mathrm{O} 1-\mathrm{Li} 2^{\mathrm{v}}$ & $74.9(2)$ \\
\hline $\mathrm{O} 1-\mathrm{Ga} 1-\mathrm{O} 4-\mathrm{Li} 2$ & $-140.21(19)$ & $\mathrm{Li} 2-\mathrm{Ga} 1-\mathrm{O} 4-\mathrm{B} 2$ & $-177.6(3)$ \\
\hline $\mathrm{O} 1-\mathrm{B} 1-\mathrm{O} 2-\mathrm{Ga}^{\mathrm{v}}$ & $-38.0(3)$ & $\mathrm{Li} 2^{\mathrm{iv}}-\mathrm{Ga} 1-\mathrm{O} 4-\mathrm{B} 2$ & $-80.7(2)$ \\
\hline $\mathrm{O} 1-\mathrm{B} 1-\mathrm{O} 2-\mathrm{Li} 2^{\mathrm{viii}}$ & $86.5(3)$ & $\mathrm{Li} 2^{\mathrm{vi}}-\mathrm{Ga} 1-\mathrm{O} 4-\mathrm{B} 2$ & $130.6(2)$ \\
\hline $\mathrm{O} 1-\mathrm{B} 1-\mathrm{O} 2-\mathrm{Li} 3^{\mathrm{ix}}$ & $-166.5(2)$ & $\mathrm{Li} 2 \mathrm{v}-\mathrm{Ga} 1-\mathrm{O} 4-\mathrm{B} 2$ & $33.0(2)$ \\
\hline $\mathrm{O} 1-\mathrm{B} 1-\mathrm{O} 3-\mathrm{Li} 1$ & $-143.3(3)$ & $\mathrm{Li}^{2}{ }^{\mathrm{iv}}-\mathrm{Ga} 1-\mathrm{O} 4-\mathrm{Li} 2$ & $96.9(2)$ \\
\hline $\mathrm{O} 1-\mathrm{B} 1-\mathrm{O} 3-\mathrm{Li} 3^{\mathrm{ii}}$ & $89.1(3)$ & $\mathrm{Li} 2{ }^{\mathrm{vi}}-\mathrm{Ga} 1-\mathrm{O} 4-\mathrm{Li} 2$ & $-51.7(2)$ \\
\hline $\mathrm{O} 1-\mathrm{B} 1-\mathrm{O} 3-\mathrm{Li3}^{\mathrm{vii}}$ & $-8.1(5)$ & $\mathrm{Li} 2 \mathrm{v}-\mathrm{Ga} 1-\mathrm{O} 4-\mathrm{Li} 2$ & $-149.4(3)$ \\
\hline $\mathrm{O} 2 \mathrm{i}-\mathrm{Ga} 1-\mathrm{O} 1-\mathrm{B} 1$ & $48.3(2)$ & 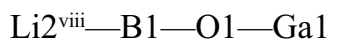 & $-145.49(15)$ \\
\hline $\mathrm{O} 2^{\mathrm{i}}-\mathrm{Ga} 1-\mathrm{O} 1-\mathrm{Li}^{\mathrm{v}}$ & $-138.71(18)$ & $\mathrm{Li} 2^{\mathrm{viii}}-\mathrm{B} 1-\mathrm{O} 1-\mathrm{Li} 2^{\mathrm{v}}$ & $43.1(4)$ \\
\hline $\mathrm{O} 2 \mathrm{i}-\mathrm{Ga} 1-\mathrm{O} 4-\mathrm{B} 2$ & $166.3(2)$ & $\mathrm{Li} 2^{\text {viii }}-\mathrm{B} 1-\mathrm{O} 2-\mathrm{Ga}^{\mathrm{v}}$ & $-124.5(2)$ \\
\hline $\mathrm{O} 2 \mathrm{i}-\mathrm{Ga} 1-\mathrm{O} 4-\mathrm{Li} 2$ & $-16.1(2)$ & $\mathrm{Li} 2^{\text {viii }}-\mathrm{B} 1-\mathrm{O} 2-\mathrm{Li}^{3}{ }^{\mathrm{ix}}$ & $107.0(2)$ \\
\hline $\mathrm{O} 2-\mathrm{B} 1-\mathrm{O} 1-\mathrm{Ga} 1$ & $165.44(16)$ & $\mathrm{Li} 2$ viii-B1-O3-Li1 & $-14.5(3)$ \\
\hline
\end{tabular}




\begin{tabular}{|c|c|c|c|}
\hline $\mathrm{O} 2-\mathrm{B} 1-\mathrm{O} 1-\mathrm{Li}^{\mathrm{v}}$ & $-5.9(4)$ & 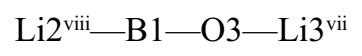 & $120.7(3)$ \\
\hline $\mathrm{O} 2-\mathrm{B} 1-\mathrm{O} 3-\mathrm{Li} 1$ & $38.4(4)$ & $\mathrm{Li} 2^{\text {viii }}-\mathrm{B} 1-\mathrm{O} 3-\mathrm{Li} 3^{\mathrm{ii}}$ & $-142.1(2)$ \\
\hline $\mathrm{O} 2-\mathrm{B} 1-\mathrm{O} 3-\mathrm{Li}^{\mathrm{ii}}$ & $-89.2(3)$ & $\mathrm{Li} 2$ - $-\mathrm{B} 2-\mathrm{O} 4-\mathrm{Ga} 1$ & $-34.6(2)$ \\
\hline $\mathrm{O} 2-\mathrm{B} 1-\mathrm{O} 3-\mathrm{Li}^{3 \mathrm{vi}}$ & $173.6(3)$ & $\mathrm{Li} 2 \mathrm{v}-\mathrm{B} 2-\mathrm{O} 4-\mathrm{Li} 2$ & $147.9(3)$ \\
\hline $\mathrm{O} 3-\mathrm{B} 1-\mathrm{O} 1-\mathrm{Ga} 1$ & $-13.0(3)$ & $\mathrm{Li} 2^{\mathrm{v}}-\mathrm{B} 2-\mathrm{O} 5-\mathrm{Ga}^{\mathrm{ii}}$ & $131.7(2)$ \\
\hline $\mathrm{O} 3-\mathrm{B} 1-\mathrm{O} 1-\mathrm{Li}^{\mathrm{v}}$ & $175.7(3)$ & $\mathrm{Li} 2^{\mathrm{v}}-\mathrm{B} 2-\mathrm{O} 5-\mathrm{Li}^{\mathrm{x}}$ & $-100.3(3)$ \\
\hline $\mathrm{O} 3-\mathrm{B} 1-\mathrm{O} 2-\mathrm{Ga}^{\mathrm{v}}$ & $140.4(2)$ & $\mathrm{Li} 2^{\mathrm{v}}-\mathrm{B} 2-\mathrm{O} 6-\mathrm{Li}^{\mathrm{xi}}$ & $-151.7(3)$ \\
\hline $\mathrm{O} 3-\mathrm{B} 1-\mathrm{O} 2-\mathrm{Li} 2^{\mathrm{viii}}$ & $-95.1(3)$ & $\mathrm{Li} 2^{\mathrm{v}}-\mathrm{B} 2-\mathrm{O} 6-\mathrm{Li}^{\mathrm{viii}}$ & $-49.8(4)$ \\
\hline $\mathrm{O} 3-\mathrm{B} 1-\mathrm{O} 2-\mathrm{Li}^{\mathrm{ix}}$ & $12.0(3)$ & $\mathrm{Li} 2 \mathrm{v}-\mathrm{B} 2-\mathrm{O} 6-\mathrm{Li} 3$ & $83.2(4)$ \\
\hline $\mathrm{O} 4-\mathrm{Ga} 1-\mathrm{O} 1-\mathrm{B} 1$ & $171.88(18)$ & $\mathrm{Li}^{3}{ }^{\mathrm{vi}}-\mathrm{Ga} 1-\mathrm{O} 1-\mathrm{B} 1$ & $16.7(2)$ \\
\hline $\mathrm{O} 4-\mathrm{Ga} 1-\mathrm{O} 1-\mathrm{Li} 2^{\mathrm{v}}$ & $-15.1(2)$ & $\mathrm{Li}^{\mathrm{vii}}-\mathrm{Ga} 1-\mathrm{O} 1-\mathrm{Li}^{\mathrm{v}}$ & $-170.3(2)$ \\
\hline $\mathrm{O} 4-\mathrm{B} 2-\mathrm{O} 5-\mathrm{Ga} 1^{\mathrm{ii}}$ & $80.4(2)$ & $\mathrm{Li}{ }^{\mathrm{vi}}-\mathrm{Ga} 1-\mathrm{O} 4-\mathrm{B} 2$ & 162.9 \\
\hline $\mathrm{O} 4-\mathrm{B} 2-\mathrm{O} 5-\mathrm{Li}^{\mathrm{x}}$ & $-151.6(2)$ & $\mathrm{Li}^{\mathrm{vii}}-\mathrm{Ga} 1-\mathrm{O} 4-\mathrm{Li} 2$ & $-19.4(3)$ \\
\hline $\mathrm{O} 4-\mathrm{B} 2-\mathrm{O} 5-\mathrm{Li}^{\mathrm{v}}$ & $-51.3(3)$ & $\mathrm{Li} 33^{\mathrm{ii}}-\mathrm{B} 1-\mathrm{O} 1-\mathrm{Ga} 1$ & $33.9(2)$ \\
\hline $\mathrm{O} 4-\mathrm{B} 2-\mathrm{O} 6-\mathrm{Li1}^{\mathrm{viii}}$ & $78.0(3)$ & $\mathrm{Li} 3^{\mathrm{ii}}-\mathrm{B} 1-\mathrm{O} 1-\mathrm{Li}^{2}$ & $-137.4(3)$ \\
\hline $\mathrm{O} 4-\mathrm{B} 2-\mathrm{O} 6-\mathrm{Li}^{\mathrm{xi}}$ & $-23.9(4)$ & $\mathrm{Li}^{3}{ }^{\mathrm{ii}}-\mathrm{B} 1-\mathrm{O} 2-\mathrm{Ga}^{\mathrm{v}}$ & $94.3(2)$ \\
\hline $\mathrm{O} 4-\mathrm{B} 2-\mathrm{O} 6-\mathrm{Li} 3$ & $-149.0(3)$ & $\mathrm{Li} 3^{\mathrm{ii}}-\mathrm{B} 1-\mathrm{O} 2-\mathrm{Li} 2^{\mathrm{viii}}$ & $-141.2(2)$ \\
\hline $\mathrm{O} 5{ }^{\mathrm{ii}}-\mathrm{Ga} 1-\mathrm{O} 1-\mathrm{B} 1$ & $-70.85(19)$ & $\mathrm{Li}^{3}{ }^{\mathrm{ii}}-\mathrm{B} 1-\mathrm{O} 2-\mathrm{Li}^{\mathrm{ix}}$ & $-34.2(3)$ \\
\hline $\mathrm{O}^{\mathrm{ii}}-\mathrm{Ga} 1-\mathrm{O} 1-\mathrm{Li}^{\mathrm{v}}$ & $102.13(18)$ & $\mathrm{Li} 3{ }^{\mathrm{ii}}-\mathrm{B} 1-\mathrm{O} 3-\mathrm{Li} 1$ & $127.6(3)$ \\
\hline $\mathrm{O} 5$ ii $-\mathrm{Ga} 1-\mathrm{O} 4-\mathrm{B} 2$ & $-69.7(2)$ & $\mathrm{Li}^{3}{ }^{\mathrm{ii}}-\mathrm{B} 1-\mathrm{O} 3-\mathrm{Li}^{\mathrm{vii}}$ & $-97.2(3)$ \\
\hline $\mathrm{O} 5{ }^{\mathrm{ii}}-\mathrm{Ga} 1-\mathrm{O} 4-\mathrm{Li} 2$ & $107.94(19)$ & $\mathrm{Li} 3-\mathrm{B} 2-\mathrm{O} 4-\mathrm{Ga} 1$ & $139.9(3)$ \\
\hline $\mathrm{O} 5-\mathrm{B} 2-\mathrm{O} 4-\mathrm{Ga} 1$ & $-3.7(3)$ & $\mathrm{Li} 3-\mathrm{B} 2-\mathrm{O} 4-\mathrm{Li} 2$ & $-37.5(5)$ \\
\hline $\mathrm{O} 5-\mathrm{B} 2-\mathrm{O} 4-\mathrm{Li} 2$ & $178.8(2)$ & $\mathrm{Li} 3-\mathrm{B} 2-\mathrm{O} 5-\mathrm{Ga} 1^{\mathrm{ii}}$ & $-81.93(16)$ \\
\hline $\mathrm{O} 5-\mathrm{B} 2-\mathrm{O} 6-\mathrm{Li} 1^{\mathrm{vii}}$ & $-100.3(3)$ & $\mathrm{Li} 3-\mathrm{B} 2-\mathrm{O} 5-\mathrm{Li}^{\mathrm{x}}$ & $46.1(2)$ \\
\hline $\mathrm{O} 5-\mathrm{B} 2-\mathrm{O} 6-\mathrm{Li}^{\mathrm{xi}}$ & $157.8(3)$ & $\mathrm{Li} 3-\mathrm{B} 2-\mathrm{O} 5-\mathrm{Li}^{\mathrm{v}}$ & $146.4(2)$ \\
\hline $\mathrm{O} 5-\mathrm{B} 2-\mathrm{O} 6-\mathrm{Li} 3$ & $32.7(4)$ & $\mathrm{Li} 3-\mathrm{B} 2-\mathrm{O} 6-\mathrm{Li}^{\mathrm{viii}}$ & $-133.1(3)$ \\
\hline $\mathrm{O} 6-\mathrm{B} 2-\mathrm{O} 4-\mathrm{Ga} 1$ & $177.91(18)$ & $\mathrm{Li} 3-\mathrm{B} 2-\mathrm{O} 6-\mathrm{Li}^{\mathrm{xi}}$ & $125.1(4)$ \\
\hline
\end{tabular}

Symmetry codes: (i) $x+1, y, z$; (ii) $-x+1,-y+1,-z+1$; (iii) $-x+1,-y,-z+2$; (iv) $-x+2,-y+1,-z+1$; (v) $x-1, y, z$; (vi) $-x+2,-y,-z+1$; (vii) $x, y-1, z+1$; (viii) $-x+1,-y,-z+1$; (ix) $x-1, y-1, z+1$; (x) $x, y+1, z-1$; (xi) $x+1, y+1, z-1$; (xii) $-x,-y-1,-z+2$; (xiii) $-x+2,-y+1,-z$; (xiv) $-x+1,-y+2,-z$. 